\title{
Expérimentelles Studium der inneren Sekretion des Pankreas.
}

\author{
V. Mitteilung : \\ Innere Sekretion des Pankreas und N. vagus. \\ Von \\ Togo Hoshi. \\ (星 䏸) \\ (Aus Prof. T. Kumagai's medizinischer Klinik an \\ der Toloku Universität zu Sendai.)
}

INHAITSYERZEICHNIS,

Einleitung.

Kapitel I. Einfluss elektrischer Reizung des $\mathrm{N}$. vagus auf Blutzucker.

I. Versuche bei Hunden,

II. Versuche bei Kaninchen.

Kapitel II. Einfluss elektrischer Reizung des N. vagus auf das Pankreashormon im Blute.

A. Versuche bei Hunden.

1. Pankreashormon in Blute vagusgereizter Hunde.

2. Pankreashormon im Blute der Kontrollfïlle.

3. Pankreashormon im Blute desselben Hundes, das ihm por und nach Reizung des $\mathrm{N}$. vagus entnommen wurde.

B. Versuche bei Kaninchen.

1. Pankreashormon in Blute vagusgereizter Kaninchen.

2. Pankreashormon im Blute der Kontrollfälle.

3. Pankreashormon im Blute splanchnikotomierter Kaninchen.

\section{Einleitung.}

Es ist eine wohlbekannte Tatsache, dass innersekretorische Funktionen der endokrinen Organe durch das Nervensystem und zwar durch das vegetative Nervensystem reguliert werden, und die Abhängigkeit der Nebenniere vom Sympathicus ist sicher nachgewiesen.

Beim Pankreas wurde die nervöse Beeinflussung auch seit langem von vielen Forschern vermutet. Aus der Fülle von Nerven in den Präparaten 
der Langerh a ns'schen Inseln, welche Dr. Uka ${ }^{1)}$ in unserer Klinik mikroskopisch untersucht hat, ist anzunehmen, dass sie auch von den Nerven beherrscht werden.

Im Jahre 1908 behaupteten Eppinger, Falta und Rudinger ${ }^{27}$ das Vorhandensein excitosekretorischer Fasern im Vagus, aber bis heute sind nur wenige experimentelle Untersuchungen erschienen, und zwar von José M. deCorall und R. Macleod, McCormick und O'brien.

José M. de Coral13) hat im Jahre 1918 unter Leitung ron Asher die Abhängigkeit der inneren Sekretion des Pankreas vom Nervensystem bei Hunden studiert und die N. vagi als innersekretorische Nerven angesprochen.

R. Macleod, McCornick und O'brien (5) haben in Jahre 1923 in Laboratorium für Physiologie zu Toronto auch dieselbe Innervation bei Hunden und Katzen untersucht und sind zu dem Schluss gekommen, dass der rechte Vagus wabrcheinlich die Innersekretion des Pankreas kontrolliert.

Nach den oben erwähnten Untersuchungen ist es wahrscheinlich, dass die $\mathrm{N}$. vagi innersekretorische Nerven des Pankreas sind; aber es gibt keinen fraglos entscheidenden Beweis dafür, und alle bisherigen Forscher selbst haben sich auch in diesem Sinne ausgesprochen. Es ist sehr wohl möglich, dass die $\mathrm{N}$. vagi die innersekretorischen Nerven des Pankreas sind, weil die Nebenniere, welche, wie schon seit langem bekannt, gegen das Pankreashormon antagonistisch wirkendes Hormon, d. h. Adrenalin sezerniert, durch den Sympathicus allein innerviert wird.

Um diese Frage zn klären, habe ich auch einige Versuche angestellt und möchte im Folgenden die erhaltenen Resultate mitteilen.

Kapitel I.

Einfluss elektrischer Reizung des N. vagus auf Blutzucker.

I. Versuche bei Hunden.

Untersuchungsmethodik.

Ich habe meine Versuche an Hunden nach de Corall und Asher in folgender' Weise ausgeführt.

Zugrunde liegte allem das Prinzip, dass Blutzucker bei dem 'Tiere, dessen Vagi gereizt werden, sich vermindern muss, falls die Sekretion des Pankreashormons durch die Vagusreizung befördert wird, weshalb man die Beeinflussung der Vagusreizung nach der Bestimmung des Blutzuckers des Tieres konstatieren kann.

Einem 24 Stunden lang hungernden Hund wurde $1 \mathrm{mg}$ Morphium hydrochloricum pro $\mathrm{kg}$ Körpergewicht ca. 30 Minuten vor der Ope- 
ration eingespritzt. Dann wurde er auf den Fixationsapparat mit Wärmevorrichtung festgebunden und unter $\ddot{A}$ thernarkose in folgender Weise operiert. Sobald das Tier narkotisiert war, machten wir (1) Tracheotomia, (2) Vagotomia duplex am Halse, um zentrifugale Reizung auszuschliessen, (3) nach Laparotomie suchten wir die Vena portae und ätzten mit konzentriertem Phenol den oberen Teil der periportalen Gegend, um dadurch alle Nervenfasern für die Leber gänzlich zu zerstören. Bei der Ätzung bedeckten wir die Organe und Gewebe um die Portalvenen völlig mit Watte und Gaze, um dadurch die Ätzwirkung des Phenols zu verhindern. Dies war nötig, um die Mobilisation des Leberglykogens durch Nerveneinfluss zu verhindern. M. Eigne $\mathrm{r}^{\text {(i) }}$ hat nachgewiesen, dass der Reiz der Vagusfasern die Mobilisation des Leberglykogens befördert. (4) Unter künstlicher Atmung eröffneten wir den rechten unteren Brustkorb und suchten die $\mathrm{N}$. vagi, welche parallel mit dem Oesophagus verlaufen, steckten sie in die Ludw ig'sche Elektrode für tiefe Nerven, reizten sie 1020 Minuten lang mit relativ schwachem Induktionsstrom bei 6 maliger Unterbrechung pro Sekunde vermittelst des Metronoms, liessen sie auch fast die gleiche Zeit ruhen und wechselten in dieser Weise mit Reizung und Ruhe ab.

Wozu ist solch eine komplizierte Operation nötig? Sie ist erforderlich, damit die Vagi unterhalb der Abzweigung des Herzastes gereizt werden, weil der N. vagus, wie schon bekannt, Hemmungsfasern für das Herz abgibt, und der Blutzucker nach Stewart und Rogof $f^{7}$ bei dem dadurch verursachten niedrigen Blutdrucke abnehmen soll.

Wir nahmen am Ende der Reiz- od. Ruheperiode Blut aus den Ohrvenen und bestinmten den Blutzucker nach der neuen Bang'schen Mikromethode. Während des Versuches ist es nötig, dass das Tier möglichst ruhig bleibt, damit man richtige Resultate erhält.

Un das Tier im ruhigen Zustand zu halten, muss man auf die Regulierung der künstlichen Atmung, die Erhaltung der Körpertemperatur und den richtigen Verlauf der Äthernarkose, welche seicht und gleichnuässig fortgesetzt werden muss, achten.

Versuche.

Um die Resultate unserer Versuche übersichtlich darzustellen, seien die Versuche im Folgenden in Tabellen und Figuren mitgeteilt. 
Tabelle I.

(Fall 1)

Nr. IV. 3. IX. 1924. Hund ô $6 \mathrm{~kg}$.

\begin{tabular}{|c|c|c|}
\hline & Zeit & $\begin{array}{l}\text { Blutzucker } \\
(\%)\end{array}$ \\
\hline $\begin{array}{l}\text { Direkt vor der Operation (vor ca. } \\
30^{\prime} 0,06 \mathrm{~g} \text { Morph. hydrochl. } \\
\text { subkutan) }\end{array}$ & $4^{\mathrm{b}} 25^{\prime}$ nachrn. & 0,123 \\
\hline Sofort nach der Operation & $5^{\mathrm{h}} 13^{\prime}$ nachm. (Operationsdauer $\left.45^{\prime}\right)$ & 0,155 \\
\hline 1. Reizperiode & $5^{\mathrm{h}} 13^{\prime}-33^{\prime}$ nachm. $\left(20^{\prime}\right)$ & 0,094 \\
\hline 1. Ruhèperiode & $5^{13} 33^{\prime}-50^{\prime}$ nachm. $\left(17^{\prime}\right)$ & 0,081 \\
\hline 2. Reizperiode & $5^{\mathrm{b}} 50^{\prime}-6^{\mathrm{b}} 10$ nachm. $\left(20^{\prime}\right)$ & 0,075 \\
\hline 2. Ruheperiode & $6^{\mathrm{h}} 10-6^{\mathrm{h}} 25^{\prime}$ nachm. $\left(15^{\prime}\right)$ & 0,064 \\
\hline
\end{tabular}

Das Resultat obiger Tabelle kann man in nachfolgender Kurve noch anschaulicher wiedergeben.

Fig. 1.

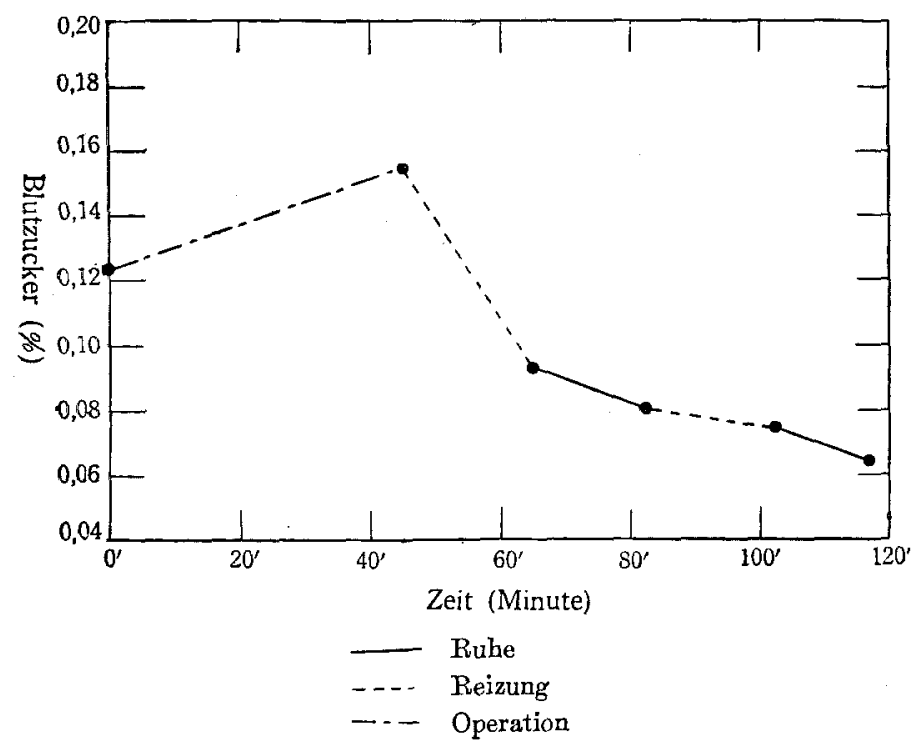

(Diese Verzeichnisse gelten auch für die sümtlichen folgenden Figuren)

Wie aus obiger Tabelle und Kurve ersichtlich nimmt der Blutzucker, der durch die 1. Reizung auffallend und rasch abgenommen hat, wührend der darauf folgenden Zeit immer mehr ab. 
Wälırend der Operation war das Tier ruhig und sah krüftig und nicht kollabiert aus. In den folgenden Versuchen werde ich mir Beschreibung des Zustandes der Tiere in jedem einzelnen Falle ersparen, abgesehen von besonders nennenswerten Zuständen.

\section{Tabelle II.}

(Fall 2)

Nr. V. 5. X. 1924. Hund ๆ 5,8 kg.

\begin{tabular}{|c|c|c|}
\hline & Zeit & $\begin{array}{c}\text { Blutzucker } \\
(\% \circ)\end{array}$ \\
\hline $\begin{array}{l}\text { Direkt vor der Operation (ror ca. } \\
30^{\prime} 0,06 \mathrm{~g} \text { Morph. hydrochl. } \\
\text { subkutan) }\end{array}$ & $10^{\mathrm{h}} 45^{\prime}$ vorm. & 0,132 \\
\hline Sofort nach der Operation & $11^{\mathrm{b}} 30^{\prime}$ vorm. (Operationsdauer $45^{\prime}$ ) & 0,143 \\
\hline 1. Reizperiode & $11^{\mathrm{h}} 30^{\prime}-50^{\prime}$ porm. $\left(20^{\prime}\right)$ & 0,082 \\
\hline 1. Ruheperiode & $11^{15} 50^{\prime}-12 /$ vorm. $\left(10^{\prime}\right)$ & 0,066 \\
\hline & $12^{\mathrm{h}}-12^{\mathrm{h}} 10^{\prime}$ nachm. $\left(10^{\prime}\right)$ & 0,063 \\
\hline 2. Reizperiode & $12^{\mathrm{b}} 10^{\prime}-23^{\prime}$ nachm. $\left(13^{\prime}\right)$ & 0,066 \\
\hline & $12^{\mathrm{h}} 23^{\prime}-35^{\prime}$ nachm. $\left(12^{\prime}\right)$ & 0,061 \\
\hline 3. Ruheperiode & $12^{\mathrm{h}} 35^{\prime}-53^{\prime}$ nachm. $\left(20^{\prime}\right)$ & 0,065 \\
\hline
\end{tabular}

Dieses Resultat kann man in einer Kurve folgendermassen darstellen.

Fig. 2.

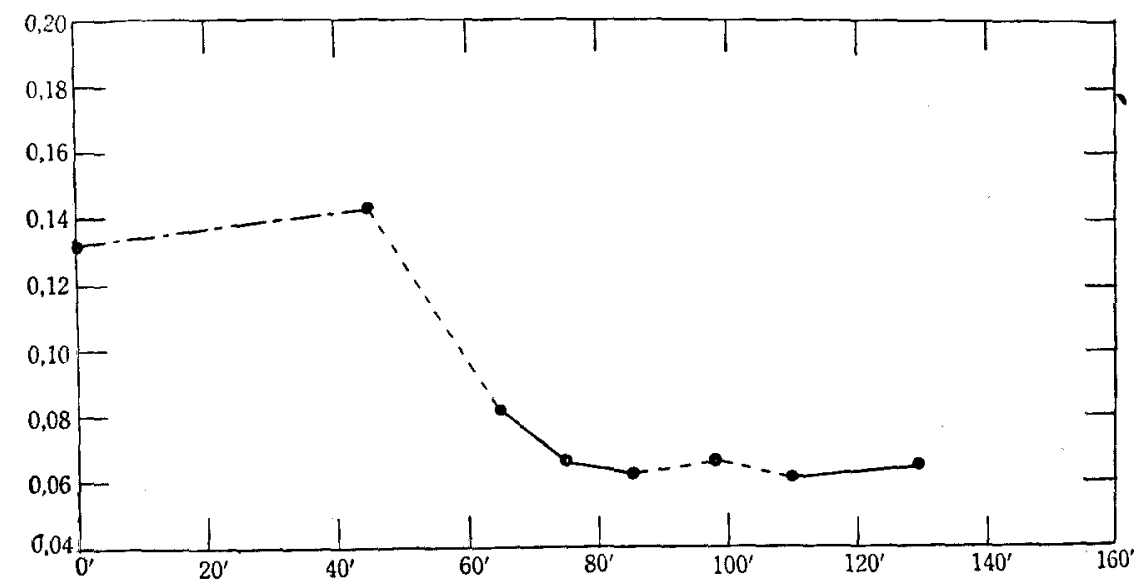

Wie aus obiger Tabelle und Kurve ersichtlich nahm der Blutzucker durch die 1. Reizung auffallend $a b$ und ist während des darauf folgenden Versuches dauernd fast unverändert geblieben. 


\section{Tabelle III.}

(Fall 3)

Nr. VI. 8. IX. 1924. Hund $\hat{s} 8,2 \mathrm{~kg}$.

\begin{tabular}{|c|c|c|}
\hline & Zeit & $\begin{array}{c}\text { Blutzucker } \\
(\%)\end{array}$ \\
\hline $\begin{array}{l}\text { Direkt vor der Operation (vor ca. } \\
\text { 30 0,08 g Morph. hydrochl. } \\
\text { subkutan) }\end{array}$ & $10^{\mathrm{h}} 37^{\prime}$ vorm. & 0,137 \\
\hline Sofort nach der Operation & $11^{\text {h } 20^{\prime}}$ vorm. (Operationsdauer $43^{\prime}$ ) & 0,196 \\
\hline & 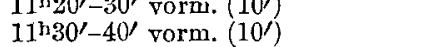 & $\begin{array}{l}0,157 \\
0,151\end{array}$ \\
\hline 1. Ruheperiode & $11^{\mathrm{h}} 40^{\prime}-50^{\prime}$ vorm. $\left(10^{\prime}\right)$ & 0,145 \\
\hline & $11^{\mathrm{b}} 50^{\prime}$ vorm. $-12^{\mathrm{n}} 5^{\prime}$ nachm. $\left(15^{\prime}\right)$ & 0,131 \\
\hline 3. Reizperiode & $12 \mathrm{~h} 5^{\prime}-20^{\prime}$ nachm. $\left(15^{\prime}\right)$ & 0,139 \\
\hline 3. Ruheperiode & $12 \mathrm{~L} 20^{\prime}-35^{\prime}$ nachm. $\left(15^{\prime}\right)$ & 0,104 \\
\hline & $12^{\mathrm{h}} 35^{\prime}-45^{\prime}$ nachm. $\left(10^{\prime}\right)$ & 0,071 \\
\hline
\end{tabular}

Aus obigen Resultaten in Tabelle III kann man eine Kurve wie unten bezeichnen.

Fig. 3.

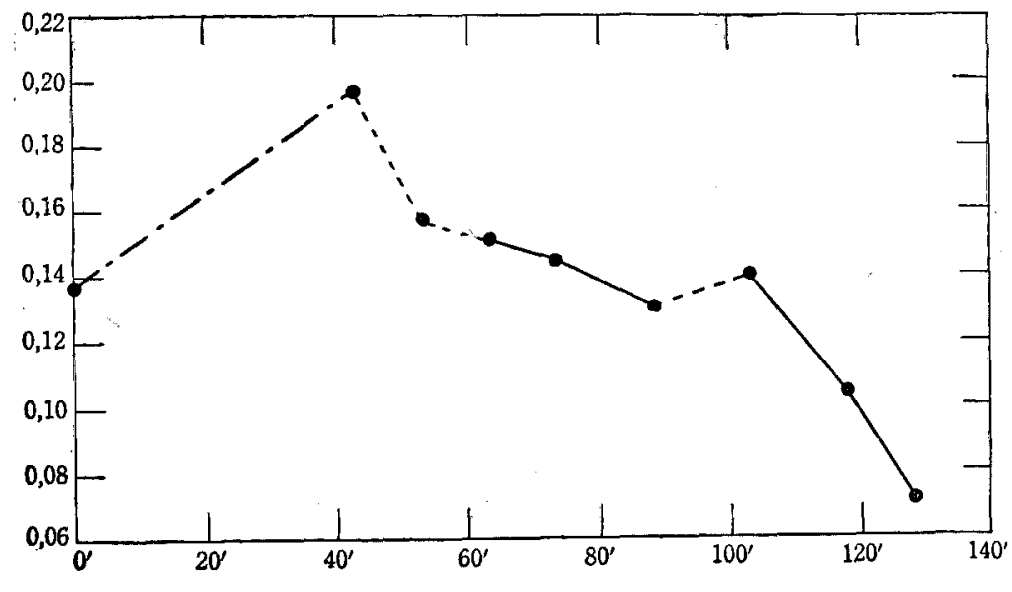

In diesem Falle nahm der Blutzucker erst durch die zweimal fortgesetzt ausgeübte Reizung mehr und mehr bis zum Ende des Versuches ab, wenn er sich auch am Ende der 3. Reizung wieder in leichtem Grad vermehrte. 
Tabelle IV.

(Fall 4)

Nr. X. 25. XI. 1924. Hund \& $12 \mathrm{~kg}$.

\begin{tabular}{|c|c|c|}
\hline & Zeit & $\begin{array}{l}\text { Blutzucker } \\
\qquad \%)\end{array}$ \\
\hline $\begin{array}{l}\text { Direkt vor der Operation (vor ca. } \\
30 / 0,12 \mathrm{~g} \text { Morph. hydrochlor. } \\
\text { subkutan) }\end{array}$ & $3^{\mathrm{b}}$ nachm. & 0,095 \\
\hline Sofort nach der Operation & $3^{h} 50^{\prime}$ nachm. (Operationsdauer $50^{\prime}$ ) & 0,191 \\
\hline 1. Reizperiode & $3^{\mathrm{b}} 50^{\prime}-4^{\mathrm{h}} 8^{\prime}$ nachm. $\left(18^{\prime}\right)$ & 0,179 \\
\hline & $4 \mathrm{~h}^{\prime}-20^{\prime}$ nachm. $\left(12^{\prime}\right)$ & 0,178 \\
\hline 1. Ruheperiode & $4 \mathrm{~h}^{2} 0^{\prime}-35^{\prime}$ nachm. $\left(15^{\prime}\right)$ & 0,163 \\
\hline $2 . \quad " \quad 1$ & $4 \mathrm{~h} 35^{\prime}-50^{\prime}$ nachm. $\left(15^{\prime}\right)$ & 0,161 \\
\hline 3. Reizperiode & $4 \mathrm{~h} 50^{\prime}-55^{\prime}$ nachm. $\left(15^{\prime}\right)$ & 0,133 \\
\hline 3. Ruheperiode & $4^{\mathrm{n}} 55^{\prime}-5^{\mathrm{h}} 5^{\prime}$ nachm. $\left(10^{\prime}\right)$ & 0,115 \\
\hline
\end{tabular}

Das obige Resultat in der Tahelle IV kann man in einer Kurve wie unten bezeichnen.

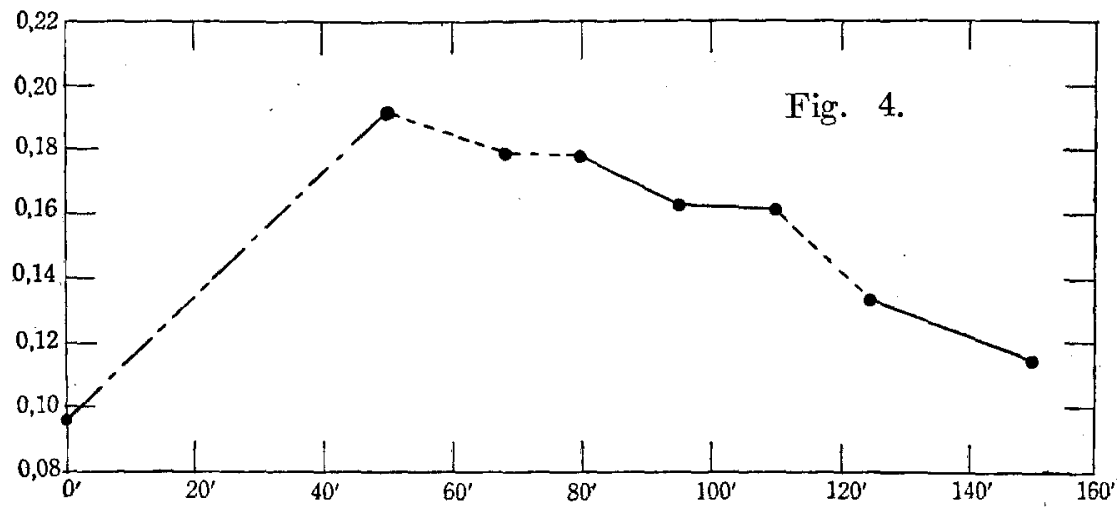

Wie aus obiger Tabelle und Kurve ersichtlich blieb der Blutzucker fast unverändert bis zur 2. Ruhe, begann aber durch die 3 . Reizung rasch und auffallend abzunehmen.

Tabelle V.

(Fall 5)

Nr. III. 2. IX. 1924. Fund $q 14,2 \mathrm{~kg}$.

\begin{tabular}{l|l|c} 
& \multicolumn{1}{|c|}{ Zeit } & $\begin{array}{c}\text { Blutzucker } \\
(\%)\end{array}$ \\
\hline Direkt vor der Operation (vor ca. & $2^{\text {h } 23^{\prime} \text { nachm. }}$ & 0,095 \\
$30^{\prime} 0,14 \mathrm{~g}$ Morph. hydrochl. & & \\
subkutan) & & \\
Sofort nach der Operation & $3^{\mathrm{h}} 20^{\prime}$ nachm. (Operationsdauer $\left.57^{\prime}\right)$ & 0,220 \\
1. Reizperiode & $3^{\mathrm{h}} 20^{\prime}-40^{\prime}$ nachm. $\left(20^{\prime}\right)$ & 0,230 \\
1. Ruheperiode & $3^{\mathrm{h}} 40^{\prime}-50^{\prime}$ nachm. $\left(10^{\prime}\right)$ & 0,240 \\
2. Reizperiode & $3^{\mathrm{h}} 50^{\prime}-4^{\mathrm{h}} 10^{\prime}$ nachm. $\left(20^{\prime}\right)$ & 0,202
\end{tabular}




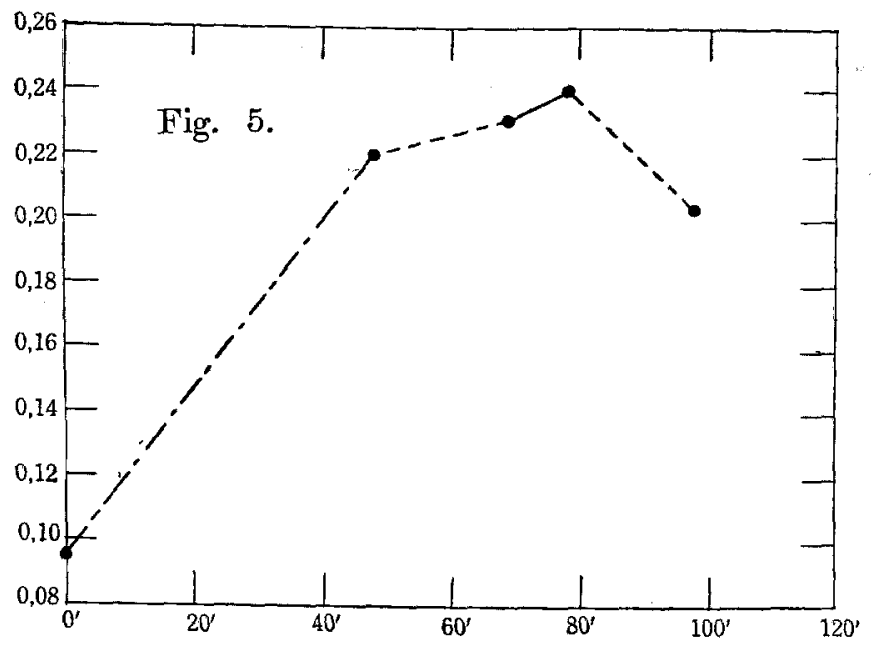

In diesem Falle nahm, wie aus obiger Tabelle und Kurve zu ersehen, der Blutzucker während der 1. Reizperiode und der direkt darauf folgenden 1. Ruheperiode zu, obgleich nur wenig, aber er begann am Ende der nächsten 2. Reizung auffallend rasch abzunehmen.

Tabelle VI.

(Fall 6)

Nr. XI. 29. IX. 1924. Hund of $16,8 \mathrm{~kg}$.

\begin{tabular}{|c|c|c|}
\hline & Zeit & $\begin{array}{c}\text { Blutzucker } \\
(\%)\end{array}$ \\
\hline $\begin{array}{l}\text { Direkt vor der Operation (vor ca. } \\
\text { 30 0,17 g Morph. hydrochl. } \\
\text { subkutan) }\end{array}$ & $10^{\mathrm{b}} 45^{\prime}$ vorm. & 0,105 \\
\hline Sofort auch der Operation & $11^{\mathrm{b}} 35^{\prime}$ vorm. (Operationsdaner 50 & 0,121 \\
\hline 1. Reizperiode & $11^{1} 35^{\prime}-50^{\prime}$ vorm. $\left(15^{\prime}\right)$ & 0,106 \\
\hline 1. Ruheperiode & $11^{\mathrm{h}} 50^{\prime}$ vorm. $-12^{\mathrm{h}} 10^{\prime}$ nachm. $\left(20^{\prime}\right)$ & 0,102 \\
\hline 2. Reizperiode & $12^{\mathrm{b}} 10^{\prime}-30^{\prime}$ nachm. $\left(20^{\prime}\right)$ & 0,111 \\
\hline 2. Ruheperiode & $12^{\mathrm{h}} 30^{\prime}-50^{\prime}$ nachm. $\left(20^{\prime}\right)$ & 0,110 \\
\hline
\end{tabular}

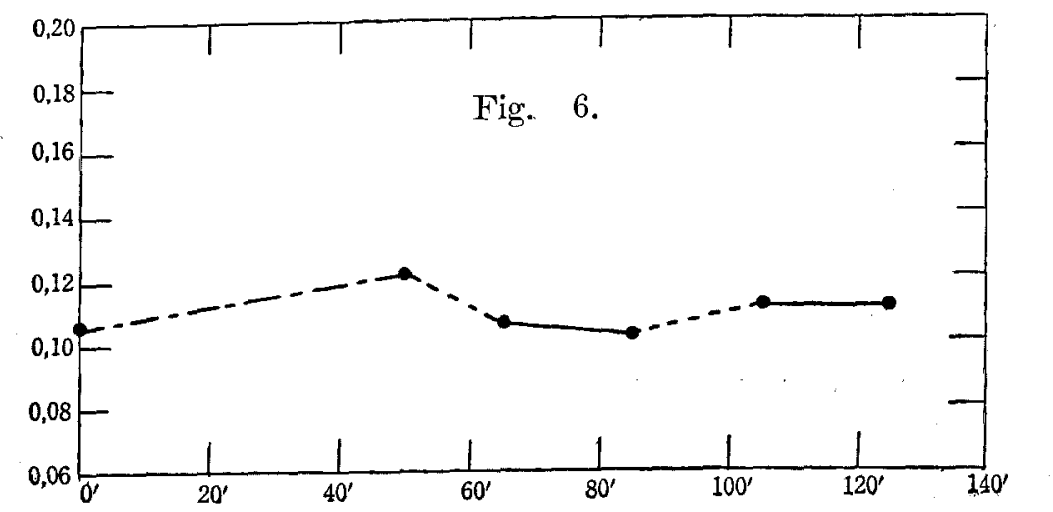


Wie obige Tabelle und Kurve zeigen, hatte der Blutzucker während des Versuches relativ leichte Bewegung, aber es besteht keine regelmässige Abhängigkeit in der Verminderung und.der Vermehrung des Blutzuckers zwischen Reizung und Ruhe.

Tabelle VII.

(Fall 7)

Nr. XIV. 21. X. 1924. Hund of $16 \mathrm{~kg}$.

\begin{tabular}{|c|c|c|}
\hline & Zeit & $\begin{array}{c}\text { Blutzucker } \\
(\%)\end{array}$ \\
\hline $\begin{array}{l}\text { Direkt vor der Operation (vor ca. } \\
30^{\prime} 0,16 \mathrm{~g} \text { Morph. hydrochl. } \\
\text { subkutan) }\end{array}$ & $10^{\mathrm{h}} 5^{\prime}$ vorm. & 0,174 \\
\hline $\begin{array}{l}\text { Sofort nach der Operation } \\
\text { 1. Reizperiode }\end{array}$ & $\begin{array}{l}10^{\mathrm{h}} 45^{\prime} \text {, vorm. (Operationsdauer } 40^{\prime} \text { ) } \\
10^{\mathrm{h}} 45^{\prime}-11^{\mathrm{l}} 2^{\prime} \text { yorm. }\left(17^{\prime}\right)\end{array}$ & $\begin{array}{l}0,268 \\
0,222\end{array}$ \\
\hline & $11^{\mathrm{h}} 2^{\prime}-15^{\prime}$ vorm. $\left(13^{\prime}\right)$ & 0,243 \\
\hline 1. Ruheperiode & $11^{\mathrm{b}} 15^{\prime}-30^{\prime}$ vorm. $\left(15^{\prime}\right)$ & 0,209 \\
\hline & $11^{\mathrm{h}} 30^{\prime}-45^{\prime}$ vorm. $\left(15^{\prime}\right)$ & 0,209 \\
\hline 3. Reizperiode & $11^{\mathrm{b}} 45^{\prime}-56^{\prime}$ vorm. (11/') & 0,240 \\
\hline 3. Rubeperiode & $11^{\mathrm{b}} 56^{\prime}$ vorm. $-12^{\mathrm{b}} 11^{\prime}$ nachm. $\left(15^{\prime}\right)$ & 0,249 \\
\hline $4 . \quad "$ & $12^{\mathrm{b}} 11^{\prime}-25^{\prime}$ nachm. $\left(14^{\prime}\right)$ & 0,240 \\
\hline
\end{tabular}

Aus obigem Resultat kann man eine Kurve wie unten bezeichnen.

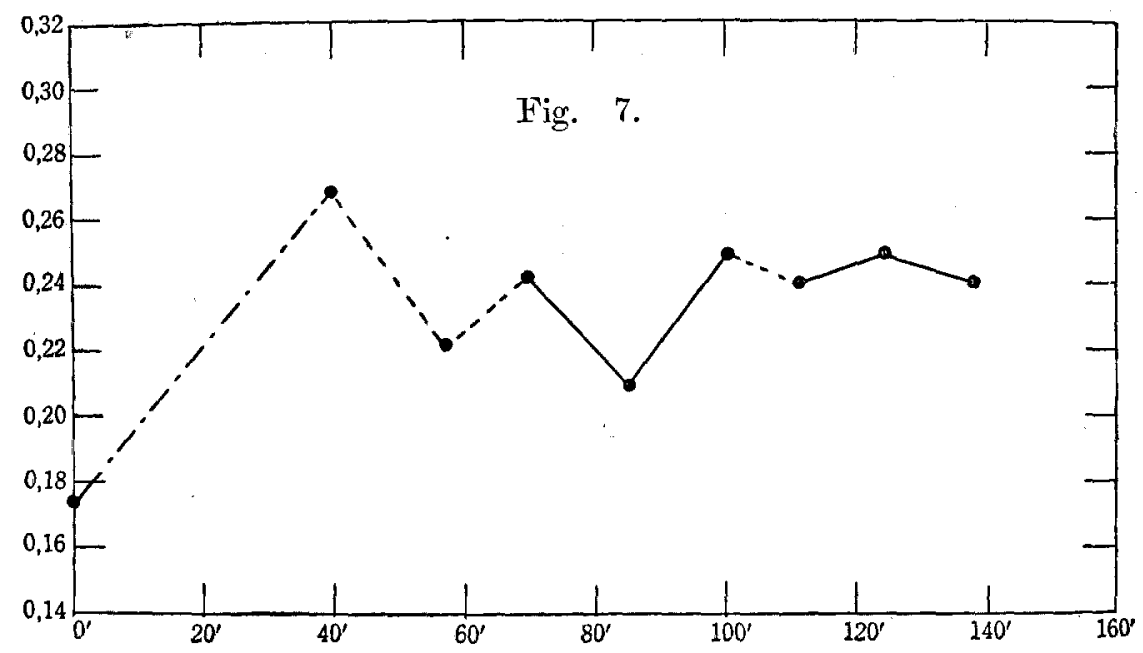

In diesem wie im vorigen Fall zeigte Blutzucker ziemlich grosse Schwankungen, ohne entsprechende regelmüssige Abhängigkeit zwischen Reizung und Ruhe. 
Tabelle VIII.

(Fall 8)

Nr. XV. 23. X. 1924. Hund of $17,6 \mathrm{~kg}$.

\begin{tabular}{|c|c|c|}
\hline & Zeit & $\begin{array}{c}\text { Blutzucker } \\
(\%)\end{array}$ \\
\hline $\begin{array}{l}\text { Direkt ror der Operation (vor ca. } \\
30^{\prime} 0,18 \mathrm{~g} \text { Morph. hydrochl. } \\
\text { subkutan.) }\end{array}$ & $2 \mathrm{~b} 25^{\prime}$ nachm. & 0,106 \\
\hline $\begin{array}{l}\text { Sofort nach der Operation } \\
\text { 1. Reizperiode } \\
\text { 1. Ruheperiode }\end{array}$ & $\begin{array}{l}\left.3^{\mathrm{h}} 5^{\prime} \text { nachm. (Operationsdauer } 40^{\prime}\right) \\
3^{\mathrm{h}} 5^{\prime}-20^{\prime} \text { nachm. }\left(15^{\prime}\right) \\
3^{\mathrm{h}} 20^{\prime}-35^{\prime} \text { nachm. }\left(15^{\prime}\right)\end{array}$ & $\begin{array}{l}0,122 \\
0,104 \\
0,104\end{array}$ \\
\hline $\begin{array}{ll}2 . & \quad 1 \\
3 . & \end{array}$ & $3^{\mathrm{h}} 35^{\prime}-50^{\prime}$ nachm. $\left(15^{\prime}\right)^{\prime}$ & 0,085 \\
\hline 2. Reizperiode & $\begin{array}{l}3^{\mathrm{n}} \mathrm{bO}^{\prime}-4^{\mathrm{n}} 30^{\prime} \text { nachm. }\left(40^{\prime}\right) \\
4^{\mathrm{s}}-40^{\prime} \text { nachm. }\left(10^{\prime}\right)\end{array}$ & $\begin{array}{l}0,126 \\
0,101\end{array}$ \\
\hline
\end{tabular}

Das Resultat kann man in eine Kurve wie unten eintragen.

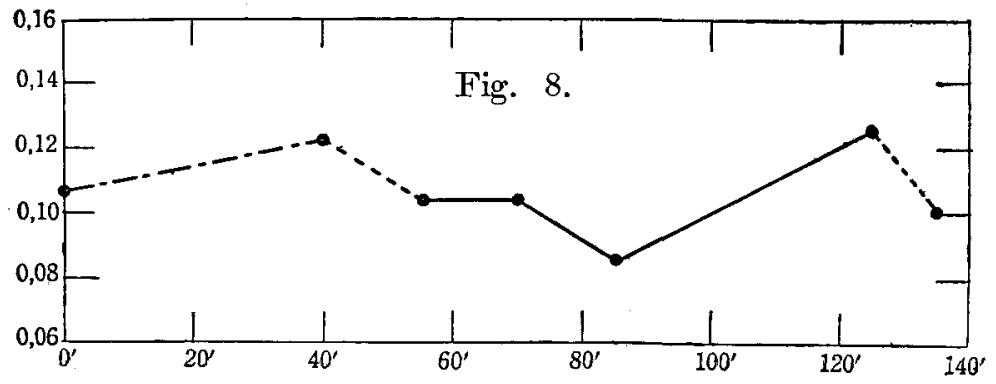

In diesem Falle beobachtete ich den Blutzucker wührend der 70 Minuten langen Ruheperiode nach der ersten, 15 Minuten langen Reizung und fand, wie aus der Tabelle und Kurve ersichtlich ist, ziemlich grosse Vermehrung am Ende der Ruheperiode, aber leider war es nicht sicher zu entscheiden, ob sie eine Folge des Zeitverlaufes oder der Unruhe des Tieres war, das gegen Ende der Ruheperiode unruhig geworden war.

Tabelle IX.

(Fall 9)

Nr. VIII. 13. IX. 1924. Hund of $8 \mathrm{~kg}$.

\begin{tabular}{|c|c|c|}
\hline & Zeit & $\begin{array}{c}\text { Blutzucker } \\
(\% 0)\end{array}$ \\
\hline $\begin{array}{l}\text { Direkt vor der Operation (ror ca. } \\
30^{\prime} 0,08 \mathrm{~g} \text { Morph. hydrochl. } \\
\text { subkutan) }\end{array}$ & $2^{\mathrm{h}}$ nachm. & 0,115 \\
\hline $\begin{array}{l}\text { Sofort nach der Operation } \\
\text { 1. Reizperiode } \\
\text { 2. Rup" } \\
\text { 1. Ruheriode }\end{array}$ & $\begin{array}{l}\left.2^{\mathrm{h}} \text { nachm. (Operationsdauer } 50^{\prime}\right) \\
2^{\mathrm{b}} 50^{\prime}-3^{\mathrm{h}} 10^{\prime} \text { nachm. }\left(20^{\prime}\right) \\
3^{\mathrm{h}} 10^{\prime}-25^{\prime} \text { nachm. }\left(15^{\prime}\right) \\
3^{\mathrm{h}} 25^{\prime}-40^{\prime} \text { nachm. }\left(15^{\prime}\right)\end{array}$ & $\begin{array}{l}0,098 \\
0,095 \\
0,087 \\
0,092\end{array}$ \\
\hline
\end{tabular}


Aus obigem Resultat kann man eine Kurve wie unten bezeichnen.

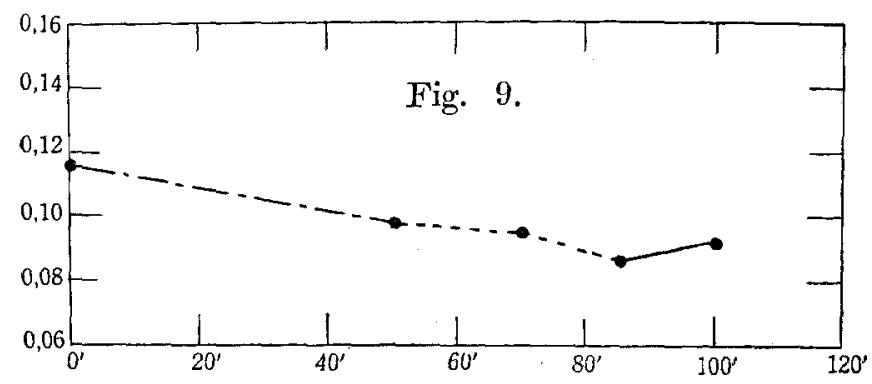

Wie aus der Tabelle und $K$ urve ersichtlich zeigte Blutzucker in diesem Falle fast keine Veränderung durch Reizung.

\section{Tabelle X. (Kontrollversuch)}

(Fall 10)

Nr. IX. 24. IX. 1924. Hund ô $10 \mathrm{~kg}$.

\begin{tabular}{|c|c|c|}
\hline & Zeit & $\begin{array}{c}\text { Blutzucker } \\
(\%)\end{array}$ \\
\hline $\begin{array}{l}\text { Direkt vor der Operation (vor } \\
\text { ca. } 30^{\prime} 0,1 \mathrm{~g} \text { Morph. hydrochl. } \\
\text { subkutan) }\end{array}$ & $11^{\mathrm{b}} 5^{\prime}$ vorm, & 0,085 \\
\hline Sofort nach der Operation & $11^{\mathrm{h}} 55^{\prime}$ vorm. (Operationsdauer $50^{\prime}$ ) & 0,098 \\
\hline $20^{\prime}$ & $11^{\mathrm{h}} 55^{\prime}$ vorm. $-12^{\mathrm{b}} 15^{\prime}$ nachm. $\left(20^{\prime}\right)$ & 0,093 \\
\hline $35 \prime$ & $12^{\mathrm{b}} 15^{\prime}-30^{\prime}$ nachm. $\left(15^{\prime}\right)$ & 0,094 \\
\hline $50^{\prime}$ & $12^{\mathrm{h}} 30^{\prime}-45^{\prime}$ nachm. $\left(15^{\prime}\right)$ & 0,087 \\
\hline $65^{\prime}$ & $12^{\mathrm{b}} 45^{\prime}-1^{\text {h }}$ nachm. $\left(15^{\prime}\right)^{\prime}$ & 0,088 \\
\hline $90^{\prime}$ & $1^{\mathrm{h}}-25^{\prime}$ nachm. $\left(25^{\prime}\right)$ & 0,078 \\
\hline
\end{tabular}

Aus obigem Resultat kann man eine Kurve wie unten bezeichnen.

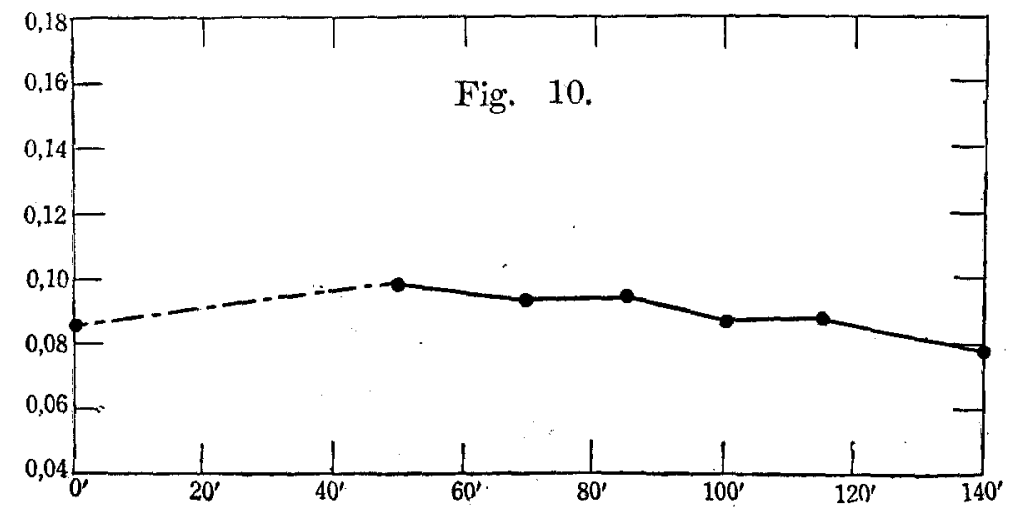


In diesem Kontrollversuche konnte man fast keine merkbare Veründerung des Blutzuckers finden.

Tabelle XI. (Kontrollversuch)

(Fall 11)

Nr. XII. 15. X. 1924. Hund \& $11,4 \mathrm{~kg}$.

\begin{tabular}{|c|c|c|}
\hline & Zeit & $\begin{array}{c}\text { Blutzucker } \\
(\%)\end{array}$ \\
\hline $\begin{array}{l}\text { Direkt vor der Operation (vor ca. } \\
30^{\prime} 0,11 \mathrm{~g} \text { Morph. hydrochl. } \\
\text { subkutan) }\end{array}$ & $2^{\mathrm{h}} 5^{\prime}$ nachm. & 0,111 \\
\hline Sofort nach der Operation & $2 \mathrm{~b} 40^{\prime}$ nachm. (Operationsdauer $85^{\prime}$ ) & 0,218 \\
\hline $20^{\prime}$ & $2^{\mathrm{l}} 40^{\prime}-3^{\mathrm{h}}$ nachm. $\left(20^{\prime}\right)$ & 0,206 \\
\hline $40^{\prime}$ & $3^{\mathrm{h}}-20^{\prime}$ nachm. $\left(20^{\prime}\right)$ & 0,208 \\
\hline $60^{\prime}$ & $3^{\mathrm{h}} 20^{\prime}-40^{\prime}$ nachm. $\left(20^{\prime}\right)$ & 0,208 \\
\hline $85^{\prime}$ & $3^{\mathrm{h}} 40^{\prime}-4^{\mathrm{h}} 5^{\prime}$ nachm. $\left(25^{\prime}\right)$ & 0,206 \\
\hline $110^{\prime}$ & $4^{\mathrm{b}} 5^{\prime}-30^{\prime}$ nachm. $\left(25^{\prime}\right)$ & 0,201 \\
\hline $125^{\prime}$ & $4^{\mathrm{h}} 30^{\prime}-45^{\prime}$ nachm. $\left(15^{\prime}\right)$ & 0,201 \\
\hline
\end{tabular}

Aus obigen Resultaten kann man eine Kurve wie unten bezeichnen.

Fig. 11.

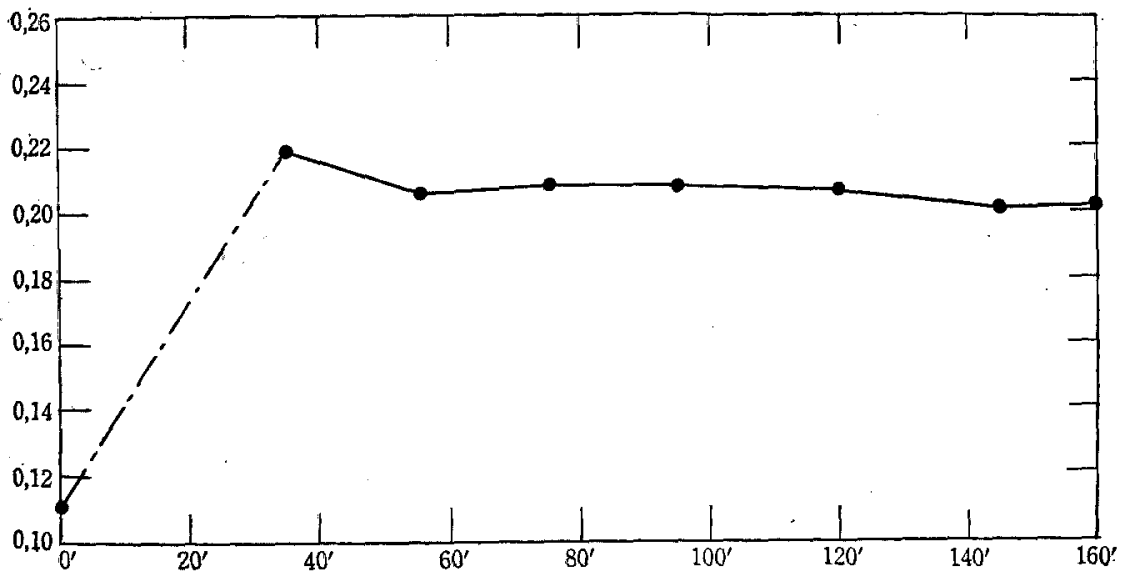

In diesem Kontrollversuche konnte man auch keine Veränderung des Blutzuckers finden. 
Tabelle XII. (Kontrollversuch)

(Fall 12)

Nr. XVI. 10. I. 1925. Hund $\delta 11 \mathrm{~kg}$.

\begin{tabular}{|c|c|c|}
\hline & Zeit & $\begin{array}{l}\text { Blutzucker } \\
(\%)\end{array}$ \\
\hline $\begin{array}{l}\text { Direkt vor der Operation (vor ca. } \\
30^{\prime} 0,11 \mathrm{~g} \text { Morph. hydrochl. } \\
\text { subkutan) }\end{array}$ & $10^{1} 40^{\prime}$ vorm. & 0,129 \\
\hline Sofort nach der Operation & $11^{\mathrm{h}} 25^{\prime}$ vorm. (Operationsdauer $45^{\prime}$ ) & 0,204 \\
\hline $15^{\prime} \quad "$ & $11^{1} 25^{\prime}-40^{\prime}$ vorm. $\left(15^{\prime}\right)$ & 0,202 \\
\hline $30^{\prime}$ & $11^{\mathrm{h}} 40^{\prime}-55^{\prime}$ vorm. $\left(15^{\prime}\right)$ & 0,194 \\
\hline $45^{\prime}$ & $11^{\mathrm{b}} 55^{\prime}$ vorm, $-12 \mathrm{~h} 10^{\prime}$ nachm. (15/) & 0,211 \\
\hline $60^{\prime}$ & $12^{\prime} 10^{\prime}-25^{\prime}$ nachm. $\left(15^{\prime}\right)$ & 0,211 \\
\hline $80^{\prime}$ & $12 \mathrm{~h}^{2} 5^{\prime}-45^{\prime}$ nachm. $\left(20^{\prime}\right)$ & 0,196 \\
\hline $85^{\prime}$ & $12^{\mathrm{h}} 45^{\prime}-1^{\mathrm{h}}$ nachm. $\left(15^{\prime}\right)$ & 0,176 \\
\hline
\end{tabular}

Aus obigem Resultaten kann man eine Kurve wie unten bezeich-] nen.

Fig. 12.

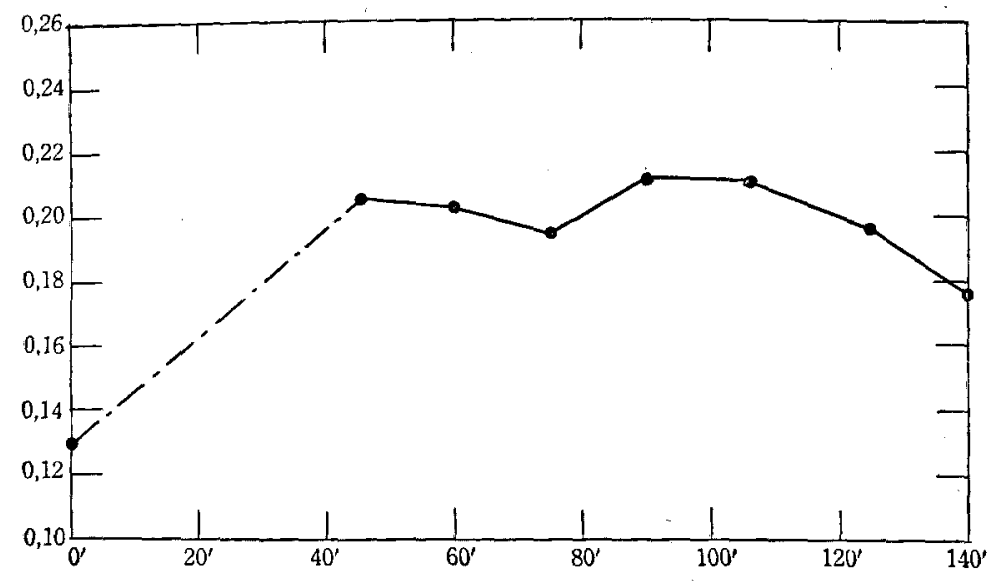

In diesem Kontrollversuche fanden wir, wie Tabelle und Kurve zeigen, ziemlich grosse Bewegung des Blutzuckers. Warum zeigte der Blntzucker solch grosse Bewegung nur bei diesem Fall? Wahrscheinlich wurde sie durch die Unruhe und die Ermüdung des Tieres verursacht, denn das Tier war während des Versuches ziemlich unruhig und kollabierte zuletzt. 


\section{Versuche bei Kaninchen.}

Bei Kaninchen wurden die Versuche mit fast gleicher Technik wie bei Hunden ausgeführt. 24 Stunden lang hungernde Kaninchen wurden am Fixationsapparat mit Wärmevorrichtung festgebunden, unter UrethanNarkose oder ohne Narkose, beide Vagi durchschnitten, laparotomiert und endlich $N$. vagus aufgesucht. Bei Kaninchen kann der untere Teil des Oesophagus in der Bauchhöhle abprïpariert werden, folglich können die ihn begleitenden Vagi auch aufgesucht werden ; sonst wird die Reiztechnik der Vagi ganz gleich wie bei Hunden ausgeführt. Bei Kaninchen sind die Resultate verschieden von denen bei Hunden; Blutzucker nahm bei allen Fällen sowie auch bei den Kontrollfällen allmählich zu, auch ganz unabhängig von der Narkose während der Untersuchung. Der Einfachheit halber werden alle Resultate zusammenfassend in Tabelle XIII wiedergegeben.

Wie aus dieser Tabelle ersichtlich, scheint die Vagusreizung keinen Einfluss auf den Blutzucker auszü̈ben. Es ist wahrscheinlich auf die Überempfindlichkeit des Adrenalsystems der Kaninchen und der dadurch hervorgerufenen lebhaften Glykogenmobilisation, schliesslich auf die versteckte Wirkung vermehrten Pankreashormons zurückzuführen, dass der Blutzucker sich nicht vermindert. Ich will dieses Problem mit anderef. Methode im folgenden Kapitel aufzuklären suchen.

\section{Zusammenfassende Betrachtung.}

Betrachtet man alle Versuche mit Hunden genauer, so kann man sie etwa in folgende 3 Gruppen einteilen.

Gruppe I. Bei dieser Gruppe nahm der Blatzucker unter dem Einfluss der Reizung im allgemeinen im Anfang auffallend rasch, dann allmählich ab. Zu dieser Gruppe gehören die Fülle Nr. IV, V, VI und X.

Bei dem Fall Nr. III zeigte der Blutzucker, wie oben beschrieben, eine leichte Vermehrung während der ersten Reizung und der ersten Ruhe, begann aber am Ende der nächstfolgenden zweiten Reizung auffallend rasch abzunehmen, jedoch konnte ich es nicht weiter beobachten, weil ich hierbei die Untersuchung wegen der Störung am Apparat einstellen musste.

Gruppe II. Bei dieser Gruppe zeigte der Blutzucker deutliche Schwankung infolge der Reizung und der Ruhe, aber die Verminderung des Blutzuckers entsprach nicht der Reizung und die Vermehrung nicht der Ruhe. Zu dieser Gruppe gehören die Fälle von Nr. XI, XIV und XV. 
Tabelle

(Fall

Blutzucker der Kaninchen, bei

\begin{tabular}{|c|c|c|c|c|c|}
\hline \multirow{2}{*}{$\begin{array}{l}\text { Vers. Nr. u. } \\
\text { Datum }\end{array}$} & \multirow{2}{*}{$\begin{array}{l}\text { Körpergew. } \\
\text { d. } \\
\text { Kaninchens }\end{array}$} & \multirow{2}{*}{$\begin{array}{l}\text { Blutzucker } \\
\text { vor d. } \\
\text { Operation }\end{array}$} & \multirow{2}{*}{$\begin{array}{c}\text { Blutzucker } \\
\text { sofort nach d. } \\
\text { Operation }\end{array}$} & \multicolumn{2}{|c|}{ Reizungsperiode } \\
\hline & & & & Zeit & Blutzucker \\
\hline 28. X. 1924 & $2300 \mathrm{~g}$ & $0,093 \%$ & $0,159 \%$ & $15^{\prime}$ & $0,136 \%$ \\
\hline 2. $\mathrm{x}^{2} \cdot 1924$ & 1800, & $0,097 ”$ & $0,179 "$ & $15^{\prime}$ & $0,205 \#$ \\
\hline 3. XI. 1924 & 1850, & $0,071 ”$ & $0,106 "$ & $15^{\prime}$ & $0,112 "$ \\
\hline 5. XI. 1924 & 2100, & $0,080 "$ & 0,186, & $20^{\prime}$ & $0,212 "$ \\
\hline 12. I. 1925 & 2500, & 0,085, & 0,116, & $12^{\prime}$ & 0,128, \\
\hline $\begin{array}{l}\text { Kontroll } \\
\quad 3 \\
\text { 3. X. } 1925\end{array}$ & $2600 "$ & $0,090 "$ & 0,100, & $15^{\prime}$ & 0,124 " \\
\hline
\end{tabular}

Gruppe III. Zu dieser Gruppe gehört nur der Fall Nr. VIII; der Blutzucker zeigte fast keine Veränderung infolge der Reizung.

Kontrollversuche. Die Kontrollfälle, bei welchen wir ausser elektrischer Reizung genau den gleichen operativen Eingriff wie bei den anderen Füllen gemacht haben, zeigten nur geringe Schwankungen des Blutzuckers während der Untersuchung, sodass man ihn unveründert nennen kann:

Nun möchte ich alle obigen Versuchsfälle zusammenfassend und kritisch betrachten.

Bei fast allen Versuchsfïllen findet man Hyperglykämie direkt nach der Operation, welche infolge der Morphium- und Äthernarkose, der Fixation und der Operation u. dgl. hervorgerufen wurde. Obgleich es sehr viele Forschungen über derartige Hyperglykämie gibt, will ich hier nicht darüber sprechen, weil der Anhaltspunkt für die Beförderung der Pankreashormonsekretion durch die Vagusreizung die Verminderung des Blutzuckers ist.

Bei Gruppe I zeigte Blutzucker auffallend rasche Abnahme, die selbstverständlich Folge des auf einmal in grosser Menge sezernierten Pankreashormons ist.

Die durch die verschiedene Behandlung verursachte Hyperglykämie kann sich nicht so rapid ausgleichen, da der Blutzucker nach Ste w art und 


\section{XIII.}

13-18)

welchen $\mathrm{N}$. vagus gereizt wurde.

\begin{tabular}{|c|c|c|c|c|c|c|}
\hline \multicolumn{2}{|c|}{ Ruheperiode } & \multicolumn{2}{|c|}{ Reizungsperiode } & \multicolumn{2}{|c|}{ Ruheperiode } & \multirow{2}{*}{ Narkose } \\
\hline Zeit & Blutzucker & Zeit & Blutzucker & Zeit & Blutzucker & \\
\hline $15^{\prime}$ & $0,120 \%$ & $20^{\prime}$ & $0,145 \%$ & $15^{\prime}$ & $0,168 \%$ & Urethan \\
\hline $25^{\prime}$ & 0,206, & $10^{\prime}$ & 0,216, & & & $"$ \\
\hline $15^{\prime}$ & $0,130 "$ & & & & & $"$ \\
\hline $17^{\prime}$ & $0,242 "$ & 7 & 0,273, & $20^{\prime}$ & $0,290 "$ & $"$ \\
\hline $10^{\prime}$ & 0,133, & $10^{\prime}$ & 0,139, & $10^{\prime}$ & $0,143 "$ & ohne \\
\hline $15^{\prime}$ & 0,123 & $15^{\prime}$ & $0,137 "$ & $15^{\prime}$ & $0,143 "$ & Urethan \\
\hline
\end{tabular}

Rogoff, wie schon erwähnt, bei der Blutdruckerniedrigung abninmt. Deshalb operierte ich das Tier so schnell wie möglich und hütete mich auch dabei möglichst vor Blutung während der Operation und vor Ermüdung des Tieres.

Bei den Fällen, welche zur Gruppe II gehören, nahm Blutzucker durch die Reizung ab, aber die Abnahme entsprach nicht der Reizung und war auch nicht so gross wie bei denen der Gruppe I; Blutzucker vermehrte sich, obgleich er einmal abgenommen hatte, während der Untersuchung wieder, aber auch nicht entsprechend der Ruheperiode. Nun wissen wir einerseits, dass die den Blutzucker herabsetzende Wirkung des Pankreashormons relativ langsam ist und in der kurzen Zeit von 10 bis 20 Minuten nicht genügend wirken kann, und dürfen deshalb anderseits vermuten, dass das durch Reizung sezernierte Pankreashormon in derselben Reizungsperiode, aber auch in der nächstfolgenden Ruheperiode noch wirksam ist.

Nach de Corall zeigte Blutzucker beim typischen Falle Verminderung entsprechend der Reizung und Vermehrung entsprechend der Ruhe; aber bei meinen Fällen war es, wie oben erwähnt, nicht so.

Nach Macleod u. a. zeigte Blutzucker auch unregelmässige Schwankungen infolge der Reizung, und sie hielten die Vermehrung des Blutzuckers für eine Folge der Ermüdung des Nerves; wenn letztere nicht vor- 
liegt, nimmt der Blutzucker nach dem Weglassen des Reizes wieder zu.

Nach meiner Meinung kann sich der Blutzucker, wenn er sich einmal bis zur Norm oder Subnorm wie bei Gr. I verminderte, nicht wieder in kurzer Zeit vermehren, aber er kann im Gegenteil, wie bei Gruppe II, wieder zunehmen, wenn das sezernierte Hormon gering und dementsprechend die Verminderung des Blutzuckers klein ist.

Zuletzt will ich noch einmal betonen, dass das Resultat stark von dem Zustand des Tieres während der Untersuchung abhängig ist; wenn es unruhig oder geschwächt ist, schwankt der Blntzucker so wie beim Fall XVI (Kontrolle), als ob der Vagus gereizt wïre.

Bei Gruppe III merkt man keinen Einfluss des Reizes auf Blutzucker, und ich hatte nur einen solchen Fall, obgleich Macleod u. a. ziemlich viele solcher Fälle gesehen haben.

Bei den Kontrollfitlen sah ich nur geringe Schwankungen des Blutzuckers, wie bei denen von de Cor all, auch bei den Fällen von Ma cleod u. a. zeigte der typische Fall ausser der anfänglichen leichten Schwankung fast keine Bewegung. Am Ende muss ich die Fälle dèr Gruppe I als typisch betrachten.

\section{Schluss.}

Aus obigen Resultaten kann nan als höchst wahrscheinlich das Vorhandensein innersekretorischer Fasern des Pankreas im N. vagus vermuten.

\section{Kapitel II.}

Einfluss elektrischer Reizung des $\mathbb{N}$. vagus auf das Pankreashormon im Blute.

Wenn die innere Sekretion des Pankreas durch N. vagus befördert wird, so kann sich Blutzucker durch Vagusreizung vermindern. Nach dieser Vermutung habe ich auch, wie de Corall und McCormick, Macleod und O'brien, eine Untersuchung, welche im Kapitel I eingehend beschrieben wurde, angestellt und bis zu einem gewissen Grad den Anhaltspunkt dafür bekommen. Aber die Beförderung hormoneller Sekretion durch Vagusreizung wurde durch Verminderung des Blutzuckers erklärt. Blutzucker ist jedoch, wie wohl bekannt, bei derartigen komplizierten Untersuchungen durch langdauernde Operation, Fesselung, Narkose und künstliche Atmung fortwährend verschiedenartig beeinflusst; deshalb kann man nicht ohne weiteres Verminderung des Blutzuckers als Folge der Vagusreizung ansehen. Besonders nimmt der Blutzucker bei Kaninchen, wie 
aus den Resultaten im vorigen Kapitel ersichtlich ist, während der Untersuchung auf Vagusreizung hin immer mehr zu. Dies wird vielleicht durch Überempfindlichkeit des Adrenalsystems des Kaninchens und folglich durch lebhafte Glykogenmobilisation verursacht. Um den Einfluss der Vagusreizung auf die innere Sekretion des Pankreas sicher zu stellen, muss man deswegen das Pankreashormon im Blute des Versuchstieres direkt messen.

Ich präparierte das Pankreashormon im Blute durch die AlkobolSchwefelsüure-Methode oder die Aceton-Pikrinsäure-Methode, über welch beide ich schon berichtet habe. Durch erstere kann man die Hälfte des enthaltenen Hormons, durch letztere fast die Gesamtmenge, wenigstens 8090\% von ihm, gewinnen. 'Ich will die Methode unten nur kurz beschreiben. Genaueres darüber kann man in meiner Veröffentlichung." "Über das Pankreashormon im Blute in verschiedenem Zustand "erfahren.

\section{Alkohol-Schwefelsäure-Methode.}

Im Verhültnis von 1,3\% setzt man dem Blut 95\% Schwefelsüure und die 3 fache Menge 95\% Alkohol zu und lässt das Gemisch 24 Stunden lang stehen; man destilliert den Alkohol und auch zum Teil das Wasser mittelst der Vakuumvorrichtung ab, dann giesst man den Rückstand in den Scheidetrichter und schüttelt ihn mit Äther; nach 12-24 Stunden entninamt man die die unterste Schicht bildende Lösung, d. h. das Extrakt durch den Hahn und verdunstet den $\ddot{A}$ ther sowie auch das Wasser so stark wie möglich in dem Faust'schen Apparat; man neutralisiert es mit verdünnter Kalilauge und spritzt es in die Unterhaut der Kaninchen. Vor und nach der Injektion bestimmt man den Blutzucker an den Kaninchen.

\section{Aceton-Pikrinsäure-Methode.}

Dies ist eine von Baker, Dickens und Dod d $\mathrm{s}^{10)}$ angegebene Methode, welche ich für die Herstellung des Pankreashormons im Blute anwandte. Man setzt $30 \mathrm{~cm}$ Blut 0,7 g Pikrinsäure in Pulverform und ca. $25 \mathrm{ccm}$ Aceton zu, mischt sie gut und zentrifugiert sie, dann dekantiert man die obenstehende klare Flüssigkeit, setzt dem Bodensatz ca. $25 \mathrm{ccm} 70 \%$ igen Acetons zu und rührt das Gemisch mit Glasstäbchen gut um, zentrifugiert und dekantiert es ; man behandelt es noch einmal mit Aq. dest. ebenso wie oben; - man vereinigt dann die oben $3 \mathrm{mal}$ dekantierten Flüssigkeiten und lässt das Aceton durch den Faust'schen Apparat austreiben; man zentrifugiert sie und giesst die obere Flüssigkeit ab; man wäscht den Bodensatz mit $\ddot{A}$ ther einige Male aus und löst ihn in 5-6 ccm Salzsäure-Alkohol, welcher aus 25 
ccm n/3 Salzsäurelösung und $75 \mathrm{ccm} 95 \%$ igem Alkohol besteht; man zentrifugiert die Lösung und dekantiert oder vielmehr saugt die obenstehende klare Flüssigkeit mit Saugpipette so vollstündig wie möglich ab, fügt ihm reines Aceton bis zur 10 fachen Menge hinzu und zentrifugiert noch einmal und dekantiert die obere Flüssigkeit, d. h. das Aceton, wäscht den Rückstand mit $\ddot{A}$ ther einige Male aus und trocknet ihn im Exsikkator auf Schwefelsäure im Vakuum. Auf diese Weise bekommt man das gewünschte Pankreashormon als weisses Pulver ; man löst das Pulver in schwach saurem Wasser ( 1 Tropfen 20 Vol. \% $\mathrm{H}_{2} \mathrm{SO}_{4} \mathrm{zu} 50 \mathrm{ccm} \mathrm{H}_{2} \mathrm{O}$ ) auf und injiziert dies in die Unterhaut eines schon 24 Stunden lang hungernden Kaninchens und bestimmt dessen Blutzucker. Wir gebrauchten die neue Bang'sche Mikromethode für die Blutzuckerbestimmung.

\section{A. Versuche bei Hunden.}

I. Pankreashormon im Blut der Hunde, bei welchen N. vagus gereizt wurde.

Die Untersuchungstechnik ist fast gleich wie die im Kapitel I geschilderte, deshalb beschreiben wir hier nur einige Hauptpunkte: bei den 24 Stunden lang hungernden Hunden wurden Tracheotomia und Vagotomia duplex ausgeführt ; unter künstlicher Atmung öffneten wir den rechten unteren Brustteil und suchten die $\mathrm{N}$. vagi auf, welche den Oesophagus begleiten; wir steckten sie in die schon erwähnte besondere Elektrode ein und reizten 10-20 Minuten lang mit relativ schwachem Induktionsstrom mit 6 maligen Unterbrechungen pro Minute mittelst des Metronoms; am Ende des Versuches entnahmen wir Blut durch die Carotiskanüle und präparierten das Pankreashormon aus dem Blut durch die oben geschilderte AlkoholSchwefelsäure-Methode; wir injizierten das gewonnene Extrakt unter die Hant der Kaninchen und bestimmten die dadurch verursachte Veränderung seines Blutzuckers.

In Folgenden seien die Hauptpunkte jedes Versuches tabellarisch mitgeteilt.

\section{Fall 19.}

Wie aus Tabelle XIV ersichtlich, nahm der Blutzucker des Hundes durch die 1. Reizung nur in geringem Grade ab ; bei der nüchsten Reizung ist er unverändert geblieben; man kann nämlich bei diesem Fall fast keinen

Einfluss der Vagusreizung auf den Blutzucker sehen. Aber das Alkohol- 
extrakt des Blutes von diesem Hunde, das am Ende der 2. Reizung, d.h. nach 40 Minuten langer Reizung, entnommen wurde, konnte den Kaninchenblutzucker auffallend, nämlich um $47 \%$, vermindern.

\section{Tabelle XIV.}

(Fall 19)

Vers. Nr. 1. 9. V. 1925. Hund क $9,7 \mathrm{~kg}$.

\begin{tabular}{|c|c|c|}
\hline & Zeit & $\begin{array}{c}\text { Blutzucker } \\
(\%)\end{array}$ \\
\hline $\begin{array}{l}\text { Direkt vor der Operation (vor } \\
\text { ca. } 30^{\prime} 0,1 \mathrm{~g} \text { Morph. hydrochl. } \\
\text { subkutan) }\end{array}$ & $10^{\mathrm{h}} 15^{\prime}$ vorm. & 0,096 \\
\hline $\begin{array}{l}\text { Sofort nach d. Operation } \\
\text { 1. Reizungsperiode } \\
\text { 2. (Blutentnahme) }\end{array}$ & $\begin{array}{l}\left.11^{\mathrm{h}} 10^{\prime} \text {, vorm. (Operationsdauer } 55^{\prime}\right) \\
11^{\mathrm{h}} 10^{\prime}-11^{\mathrm{h}} 25^{\prime} \text { vorm. }\left(15^{\prime}\right) \\
11^{\mathrm{h}} 25^{\prime}-11^{\mathrm{h}} 50^{\prime} \text { vorm. }\left(25^{\prime}\right)\end{array}$ & $\begin{array}{l}0,156 \\
0,148 \\
0,149\end{array}$ \\
\hline
\end{tabular}

Den Einfluss des Pankreashormons im obigen Hundeblute auf den Blutzucker eines Kaninchens sieht man im folgenden.

\begin{tabular}{|c|c|c|c|c|c|c|c|}
\hline \multirow[b]{2}{*}{$\begin{array}{c}\text { Menge d. } \\
\text { Blutes }\end{array}$} & \multirow{2}{*}{$\begin{array}{l}\text { Methode d. } \\
\text { Isolierung } \\
\text { d. Hormons }\end{array}$} & \multirow[b]{2}{*}{$\begin{array}{l}\text { Menge d. } \\
\text { Extraktes }\end{array}$} & \multirow{2}{*}{$\begin{array}{l}\text { Körp. } \\
\text { Gew.d. } \\
\text { Kanin. }\end{array}$} & \multicolumn{3}{|c|}{ Blutzucker $(\%)$} & \multirow{2}{*}{$\begin{array}{l}\text { Grad d. } \\
\text { Vermin- } \\
\text { derung } \\
\text { d. Blut- } \\
\text { zuckers }\end{array}$} \\
\hline & & & & $\begin{array}{l}\text { vor d. } \\
\text { Inj. }\end{array}$ & $\begin{array}{l}2 \frac{1}{2} \text { St. } \\
\text { nach d. } \\
\text { Inj. }\end{array}$ & $\begin{array}{c}4 \frac{1}{2} \text { St. } \\
\text { nach d. } \\
\text { Inj. }\end{array}$ & \\
\hline $140 \mathrm{cc}$ & $\begin{array}{c}\text { Alkohol- } \\
\mathrm{H}_{2} \mathrm{SO}_{4}\end{array}$ & $10 \mathrm{ccm}$ & $1280 \mathrm{~g}$ & 0,104 & 0,056 & 0,071 & $47 \%$ \\
\hline
\end{tabular}

Dies ist das Verhältnis des verminderten Blutzuckers zu dem vor der Injektion.

\section{Fall 20.}

Bei diesem Fall nahm Blutzucker fast entsprechend dem Reize und der Ruhe ab oder zu, besonders verminderte er sich auffallend nach der 2. Reizung ; man kann bei diesem Fall deutlichen Einfluss der Vagusreizung auf den Blutzucker sehen. Das Alkoholextrakt des Hundeblutes konnte Kaninchenblutzucker deutlich, nämlich um $30 \%$, vermindern. 
Tabelle XV.

(Fall 20)

Vers. Nr. 2. 13. V. 1925. Hund ô $18,8 \mathrm{~kg}$.

\begin{tabular}{|c|c|c|}
\hline & Zeit & $\begin{array}{c}\text { Blutzucker } \\
(\%)\end{array}$ \\
\hline $\begin{array}{l}\text { Direkt vor der Operation (vor ca. } \\
30^{\prime} 0,19 \mathrm{~g} \text { Morph. hydroch. } \\
\text { subkutan) }\end{array}$ & $10^{\mathrm{h}} 56^{\prime}$ vorm. & 0,117 \\
\hline Sofort nach d. Operation & $11^{\mathrm{h}} 455^{\prime}$ vorm. (Operationsdauer $49^{\prime}$ ) & 0,142 \\
\hline $\begin{array}{l}\text { 1. Reizungsperiode } \\
\text { 1. Ruheperiode }\end{array}$ & $\begin{array}{l}11^{\mathrm{h}} 45^{\prime}-55^{\prime} \text { vorm. }\left(10^{\prime}\right) \\
11^{\mathrm{b}} 55^{\prime} \text { vorm. }-12 \mathrm{~b}^{\prime} 5^{\prime} \text { nachm. }\left(20^{\prime}\right)\end{array}$ & $\begin{array}{l}0,136 \\
0,152\end{array}$ \\
\hline $\begin{array}{l}\text { 2. Reizungsperiode } \\
\text { (Blutentnahme) }\end{array}$ & $12^{\mathrm{b} 15^{\prime}-12^{\mathrm{b}} 30^{\prime}}$ nachm. $\left(15^{\prime}\right)$ & 0,106 \\
\hline
\end{tabular}

Der Finfluss des Pankreashormons im obigen Hundeblute auf den Blutzucker eines Kaninchens wird im folgenden gezeigt.

\begin{tabular}{|c|c|c|c|c|c|c|c|}
\hline \multirow[b]{2}{*}{$\begin{array}{c}\text { Menge d. } \\
\text { Blutes }\end{array}$} & \multirow{2}{*}{$\begin{array}{l}\text { Methode d. } \\
\text { Isolierung } \\
\text { d. Hormons }\end{array}$} & \multirow[b]{2}{*}{$\begin{array}{l}\text { Menge d. } \\
\text { Extraktes }\end{array}$} & \multirow{2}{*}{$\begin{array}{l}\text { Körp. } \\
\text { Gew. d. } \\
\text { Kanin. }\end{array}$} & \multicolumn{3}{|c|}{ BJutzucker $(\%)$} & \multirow{2}{*}{$\begin{array}{l}\text { Grad d. } \\
\text { Vermin- } \\
\text { derung } \\
\text { d. Blut- } \\
\text { zuckers }\end{array}$} \\
\hline & & & & $\begin{array}{l}\text { vor d. } \\
\text { Inj. }\end{array}$ & $\begin{array}{l}2 \frac{1}{2} \mathrm{St} . \\
\text { nach d. } \\
\text { Inj. }\end{array}$ & $\begin{array}{l}4 \frac{1}{2} \text { St. } \\
\text { nach d. } \\
\text { Inj. }\end{array}$ & \\
\hline $140 \mathrm{cc}$ & $\begin{array}{l}\text { AIkohol- } \\
\mathrm{I}_{2} \mathrm{SO}_{4}\end{array}$ & $9 \mathrm{ccm}$ & $1470 \mathrm{~g}$ & 0,102 & 0,090 & 0,071 & $30 \%$ \\
\hline
\end{tabular}

Fall 21.

Bei diesem Fall nahm Blutzucker anfangs anf das Reizen hin vielmehr zu, sank dann aber in der darauffolgenden Ruheperiode. Das Alkoholextrakt des Hundeblutes konnte Kanichenblutzucker deutlich, nämlich um $26 \%$, vermindern.

\section{Tabelle XVI.}

(Fall 21)

Vers. Nr. 4. 20. V. 1925. Hund $\circ 6,5 \mathrm{~kg}$.

\begin{tabular}{|c|c|c|}
\hline & Zeit & $\begin{array}{c}\text { Blutzucker } \\
(\%)\end{array}$ \\
\hline $\begin{array}{l}\text { Direkt vor der Operation (vor } \\
\text { ca. } 30^{\prime} 0,7 \mathrm{~g} \text { Morph. hydrochl. } \\
\text { subkutan) }\end{array}$ & $10^{\mathrm{h}} 30^{\prime}$ vorm. & 0,104 \\
\hline Sofort nach d. Operation & $\begin{array}{l}11^{\mathrm{b}} 18^{\prime} \text { vorm. (Operationsdauer } 48^{\prime} \text { ) } \\
11^{\mathrm{b}} 18^{\prime}-35^{\prime} \text { - }\left(17^{\prime}\right)\end{array}$ & $\begin{array}{l}0,152 \\
0,184\end{array}$ \\
\hline 1. Ruheperiode & $11^{\mathrm{b}} 35^{\prime}-53^{\prime}$ vorm. $\left(18^{\prime}\right)$ & 0,171 \\
\hline 2. (Blutentrahme) & $11^{15} 53^{\prime}$ vorm.-12h8' nachm. $\left(15^{\prime \prime}\right)$ & 0,159 \\
\hline
\end{tabular}


Der Einfluss des Pankreashormons im obigen Hundeblute auf den Blutzucker eines Kaninchens ist wie folgt.

\begin{tabular}{c|c|c|c|c|c|c|c}
\hline $\begin{array}{c}\text { Menge d. } \\
\text { Blutes }\end{array}$ & $\begin{array}{c}\text { Methode d. } \\
\text { Isolierung } \\
\text { d. Hormons }\end{array}$ & $\begin{array}{c}\text { Menge d. } \\
\text { Extraktes }\end{array}$ & $\begin{array}{c}\text { Körp. } \\
\text { Gew. d. } \\
\text { Kanin. }\end{array}$ & $\begin{array}{c}\text { vor d. } \\
\text { Inj. }\end{array}$ & $\begin{array}{c}2 \frac{1}{2} \text { St. } \\
\text { nach d. } \\
\text { Inj. }\end{array}$ & $\begin{array}{c}\text { Blutzucker }(\%) \\
\text { nach d. } \\
\text { Inj. }\end{array}$ & $\begin{array}{c}\text { Grad d. } \\
\text { Vermin- } \\
\text { derung } \\
\text { zuckers }\end{array}$ \\
\hline $140 \mathrm{ccm}$ & $\begin{array}{c}\text { Alkohol- } \\
\mathrm{H}_{2} \mathrm{SO}_{4}\end{array}$ & $11 \mathrm{cem}$ & $1500 \mathrm{~g}$ & 0,092 & 0,069 & 0,090 & $26 \%$ \\
\hline
\end{tabular}

Fall 22.

Bei diesem Fall nahm Blutzucker durch Vagusreizung ab ; das Alkoholextrakt dieses Hundeblutes konnte Kaninchenblutzucker um 32\%, vermindern.

\section{Tabelle XVII.}

(Fall 22)

Vers. Nr. 7. 6. V. 1925 . Hund o $8,5 \mathrm{~kg}$.

\begin{tabular}{|c|c|c|}
\hline & Zeit & $\begin{array}{c}\text { Blutzucker } \\
(\%)\end{array}$ \\
\hline $\begin{array}{l}\text { Direkt vor der Operation (vor ca. } \\
30^{\prime} 0,09 \mathrm{~g} \text { Morph. hydrochl. } \\
\text { subkutan) }\end{array}$ & $9^{\mathrm{b}} 25^{\prime}$ vorm. & 0,087 \\
\hline Sofort nach d. Operation & $10^{\mathrm{b}} 23^{\prime}$ vorm. (Operationsdauer $58^{\prime}$ ) & 0,137 \\
\hline 1. Reizungsperiode & 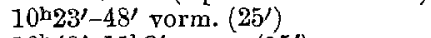 & 0,112 \\
\hline 1. Ruheperiode & $10^{\mathrm{h}} 48^{\prime}-11^{\mathrm{h}} 3^{\prime}$ vorm. $\left(15^{\prime}\right)$ & $\overline{-}$ \\
\hline $\begin{array}{l}\text { 2. Reizungsperiode } \\
\text { (Blutentnahme) }\end{array}$ & $11^{\mathrm{h}} 3^{\prime}-8^{\prime}$ vorm. $\left(5^{\prime}\right)$ & 0,010 \\
\hline
\end{tabular}

Der Einfluss des Pankreashormons im obigen Hundeblute auf den Blutzucker eines Kaninchens ist im folgenden gezeigt.

\begin{tabular}{|c|c|c|c|c|c|c|c|}
\hline \multirow[b]{2}{*}{$\begin{array}{l}\text { Menge } d . \\
\text { Blutes }\end{array}$} & \multirow[b]{2}{*}{$\begin{array}{l}\text { Methode d. } \\
\text { Isolierung } \\
\text { d. Hormons }\end{array}$} & \multirow[b]{2}{*}{$\begin{array}{l}\text { Menge d. } \\
\text { Extraktes }\end{array}$} & \multirow[b]{2}{*}{$\begin{array}{l}\text { Körp. } \\
\text { Gew. d. } \\
\text { Kanin. }\end{array}$} & \multicolumn{3}{|c|}{ Blutzucker $(\%)$} & \multirow{2}{*}{$\begin{array}{l}\text { Grad d. } \\
\text { Vermin- } \\
\text { derung } \\
\text { d. Blut- } \\
\text { zuckers }\end{array}$} \\
\hline & & & & $\begin{array}{l}\text { vor d. } \\
\text { Inj. }\end{array}$ & $\begin{array}{l}2 \frac{\mathrm{St}}{2} \text {. } \\
\text { Iach d. } \\
\text { Inj. }\end{array}$ & $\begin{array}{c}4 \frac{1}{2} \mathrm{St} . \\
\text { nach d. } \\
\text { Inj. }\end{array}$ & \\
\hline $140 \mathrm{ccm}$ & $\begin{array}{c}\text { Alkohol- } \\
\mathrm{H}_{2} \mathrm{SO}_{4}\end{array}$ & $10 \mathrm{ccm}$ & $1600 \mathrm{~g}$ & 0,090 & 0,062 & 0,078 & $32 \%$ \\
\hline
\end{tabular}

Fall 23.

Bei diesem Fall nahm Blutzucker auch durch Vagusreizung ab; aber 
das Alkoholextrakt dieses Hundeblutes konnte das Niveau des Kaninchenblutzuckers nicht deutlich herabsetzen.

Tabelle XVIII.

(Fall 23)

Vers. Nr. 8. 25. V. 1925. Hund o $8,5 \mathrm{~kg}$.

\begin{tabular}{|c|c|c|}
\hline & Zeit & $\begin{array}{c}\text { Blutzucker } \\
(\%)\end{array}$ \\
\hline $\begin{array}{l}\text { Direkt ror der Operation (vor ca. } \\
30^{\prime} 0,09 \mathrm{~g} \text { Morph. hydrochl. } \\
\text { subkutan) }\end{array}$ & $10^{\mathrm{h}} 10^{\prime}$ vorm. & 0,108 \\
\hline Sofort nach d. Operation & $10^{\mathrm{h}} 55^{\prime}$ vorm. (Operationsdauer $50^{\prime}$ ) & 0,111 \\
\hline 1. Reizungsperiode & $10^{\mathrm{h}} 55^{\prime}-11^{\mathrm{b}} 10^{\prime}$ vorm. $\left(15^{\prime}\right)$ & 0,089 \\
\hline 2. & $11^{\mathrm{h}} 10^{\prime}-25^{\prime}$ vorm. $\left(15^{\prime}\right)$ & - \\
\hline $\begin{array}{l}\text { 1. Ruheperiode } \\
\text { (Blutentnahme) }\end{array}$ & $11^{\prime} 25^{\prime}-30^{\prime}$ vorm. $\left(5^{\prime}\right)$ & 0,079 \\
\hline
\end{tabular}

Den Einfluss des Pankreashormons im obigen Hundeblut auf den Blutzucker eines Kaninchens findet man in folgender Tabelle.

\begin{tabular}{|c|c|c|c|c|c|c|c|}
\hline \multirow[b]{2}{*}{$\begin{array}{l}\text { Menge d. } \\
\text { Blutes }\end{array}$} & \multirow[b]{2}{*}{$\begin{array}{l}\text { Methode d. } \\
\text { Isolierung } \\
\text { d. Hormons }\end{array}$} & \multirow[b]{2}{*}{$\begin{array}{l}\text { Menge d. } \\
\text { Extraktes }\end{array}$} & \multirow[b]{2}{*}{$\begin{array}{l}\text { Körp. } \\
\text { Gew. d. } \\
\text { Kanin. }\end{array}$} & \multicolumn{3}{|c|}{ Blutzucker $(\%)$} & \multirow{2}{*}{$\begin{array}{l}\text { Grad d. } \\
\text { Vermin- } \\
\text { derung } \\
\text { d. Blut- } \\
\text { zuckers }\end{array}$} \\
\hline & & & & $\begin{array}{l}\text { vor d. } \\
\text { Inj. }\end{array}$ & $\begin{array}{c}2 \frac{1}{2} \mathrm{St} . \\
\text { nach d. } \\
\text { Inj. }\end{array}$ & $\begin{array}{l}4 \frac{1}{2} \mathrm{St} . \\
\text { nach d. } \\
\text { Inj. }\end{array}$ & \\
\hline $140 \mathrm{ccm}$ & $\begin{array}{c}\text { Alkohol- } \\
\mathbf{H}_{2} \mathrm{SO}_{4}\end{array}$ & $9 \mathrm{ccm}$ & $1400 \mathrm{~g}$ & 0,089 & 0,089 & 0,104 & $\begin{array}{l}\text { fust un- } \\
\text { veründert. }\end{array}$ \\
\hline
\end{tabular}

II. Pankreashormon im Blute der Hunde, bei welchen zur Kontrolle N. vagus nicht gereizt wurde.

Bei diesen Kontrollfällen haben wir ausser elektrischer Reizung ganz den gleichen operativen Eingriff wie bei den vorigen Fällen gemacht. Wir teilen unten tabellarisch die wichtigsten Punkte jedes Versuches mit.

\section{Fall 24.}

Bei diesem Fall stieg das Niveau des Blutzuckers während der Untersuchung, und das Alkoholextrakt dieses Hundeblutes konnte Kaninchenblutzucker auch nur in geringem Grad, d. h. um 7\%, vermindern. 
Tabelle XIX.

(Fall 24)

Vers. Nr. 3. 16. V. 1925. Hund of $10,5 \mathrm{~kg}$.

\begin{tabular}{|c|c|c|}
\hline & Zeit & $\begin{array}{l}\text { Blutzucker } \\
(\%)\end{array}$ \\
\hline $\begin{array}{l}\text { Direkt vor d. Operation (vor ca. } \\
30^{\prime} 0,11 \mathrm{~g} \text { Morph. hydrochl. } \\
\text { subkutan) }\end{array}$ & $9^{b} 55^{\prime}$ vorm. & 0,092 \\
\hline $\begin{array}{l}\text { Sofort nach d. Operation } \\
45^{\prime} \text { nach d. Operation } \\
\text { (Blutentnahme) }\end{array}$ & $\begin{array}{l}\left.\text { 10h35' } 75^{\prime} \text { vorm. (Operationsdauer } 43^{\prime}\right) \\
10^{\mathrm{h}} 38^{\prime}-11^{\mathrm{h}} 18^{\prime} \text { vorm. }\left(45^{\prime}\right)\end{array}$ & $\begin{array}{l}0,125 \\
0,131\end{array}$ \\
\hline
\end{tabular}

Der Einfluss des Pankreashormons im obigen Hundeblute auf den Blutzucker eines Kaninchens ist wie folgt.

\begin{tabular}{|c|c|c|c|c|c|c|c|}
\hline \multirow[b]{2}{*}{$\begin{array}{l}\text { Menge d. } \\
\text { Blutes }\end{array}$} & \multirow[b]{2}{*}{$\begin{array}{l}\text { Methode d. } \\
\text { Isolierung } \\
\text { d. Hormons }\end{array}$} & \multirow[b]{2}{*}{$\begin{array}{l}\text { Menge d. } \\
\text { Extraktes }\end{array}$} & \multirow[b]{2}{*}{$\begin{array}{l}\text { Körp. } \\
\text { Gew. d. } \\
\text { Kanin. }\end{array}$} & \multicolumn{3}{|c|}{ Blutzucker $(\%)$} & \multirow{2}{*}{$\begin{array}{l}\text { Grad d. } \\
\text { Vermin } \\
\text { derung } \\
\text { d. Blut- } \\
\text { zuckers }\end{array}$} \\
\hline & & & & $\begin{array}{l}\text { vor d. } \\
\text { Inj. }\end{array}$ & $\begin{array}{l}2 \frac{1}{2} \text { St. } \\
\text { nach d. } \\
\text { Inj. }\end{array}$ & $\begin{array}{l}4 \frac{1}{2} \text { St. } \\
\text { nach d. } \\
\text { Inj. }\end{array}$ & \\
\hline $140 \mathrm{ccm}$ & $\begin{array}{l}\text { Alkohol- } \\
\mathrm{H}_{2} \mathrm{SO}_{4}\end{array}$ & $12 \mathrm{ccm}$ & $1320 \mathrm{~g}$ & 0,090 & 0,084 & 0,086 & $7 \%$ \\
\hline
\end{tabular}

Fall 25.

Auch bei diesem Fall ist Blutzucker während der Untersuchung fast unveründert geblieben, und das Alkoholextrakt des Hundeblutes konnte Kaninchenblutzucker nur um 6\%, vermindern.

Tabelle XX.

(Fall 25)

Vers. Nr. 5. 22. V. 1925. Hund o $17 \mathrm{~kg}$.

\begin{tabular}{|c|c|c|}
\hline & Zeit & $\begin{array}{l}\text { Blutzucker } \\
\quad(\%)\end{array}$ \\
\hline $\begin{array}{l}\text { Direkt vor der Operation (vor ca. } \\
30 / 0,17 \mathrm{~g} \text { Morph. hydrochl. }\end{array}$ & $9^{\mathrm{h}} 55^{\prime}$ vorin. & 0,150 \\
\hline $\begin{array}{l}\text { subkutan) } \\
\text { Sofort nach d. Operation }\end{array}$ & $\begin{array}{l}\left.10^{\mathrm{h}} 55^{\prime} \text { rorm. (Operationsdauer } 1^{\mathrm{l}}\right) \\
10^{\mathrm{h}} 55^{\prime}-11^{\mathrm{h} 20^{\prime}} \text { yorm. }\left(25^{\prime}\right)\end{array}$ & $\begin{array}{l}0,197 \\
0,194\end{array}$ \\
\hline $\begin{array}{lc}25^{\prime} & " \prime \\
45^{\prime} & \text { (Blutentnahme) }\end{array}$ & $\begin{array}{l}11^{\prime \prime} \mathrm{b}^{\prime}-11^{2} 20^{\prime} \text { vorm. }\left(20^{\prime}\right) \\
1^{\prime} 20^{\prime}-40^{\prime} \text { vorm. }\left(20^{\prime}\right)\end{array}$ & $\begin{array}{l}0,194 \\
0,190\end{array}$ \\
\hline
\end{tabular}

Der Einfluss des Pankreashormons im obigen Hundeblute auf den Blutzucker eines Kaninchens ist wie folgt. 


\begin{tabular}{|c|c|c|c|c|c|c|c|}
\hline \multirow[b]{2}{*}{$\begin{array}{l}\text { Menge d. } \\
\text { Blutes }\end{array}$} & \multirow{2}{*}{$\begin{array}{l}\text { Methode d. } \\
\text { Isolierung } \\
\text { d. Hormons }\end{array}$} & \multirow[b]{2}{*}{$\begin{array}{l}\text { Menge d. } \\
\text { Extraktes }\end{array}$} & \multirow{2}{*}{$\begin{array}{l}\text { Körp. } \\
\text { Gew. d. } \\
\text { Kanin. }\end{array}$} & \multicolumn{3}{|c|}{ Blutzucker (\%) } & \multirow{2}{*}{$\begin{array}{l}\text { Grad d. } \\
\text { Vernin- } \\
\text { derung } \\
\text { d. Blut- } \\
\text { zuckers }\end{array}$} \\
\hline & & & & $\begin{array}{l}\text { vor d. } \\
\text { Inj. }\end{array}$ & $\begin{array}{c}2 \frac{1}{2} \mathrm{St} . \\
\text { nach d. } \\
\text { Inj. }\end{array}$ & $\begin{array}{l}4 \frac{7}{2} \mathrm{St} . \\
\text { nach d. } \\
\text { Inj. }\end{array}$ & \\
\hline $140 \mathrm{ccm}$ & $\begin{array}{c}\text { Alkohol- } \\
\mathrm{H}_{2} \mathrm{SO}_{4}\end{array}$ & $12 \mathrm{ccm}$ & $1200 \mathrm{~g}$ & 0,085 & 0,081 & 0,088 & $6 \%$ \\
\hline
\end{tabular}

Fall 26.

Bei diesem Fall stieg der Blutzucker, obgleich geringfügig, während der Untersuchung, und das Alkoholextrakt des Hundeblutes konnte Kaninchenblutzucker nicht vermindern.

\section{Tabelle XXI.}

(Fall 26)

Vers. Nr.6. 23. V. 1925. Hund 8 7,5 kg.

\begin{tabular}{|c|c|c|}
\hline & Zeit & $\begin{array}{l}\text { Blutzucker } \\
(\%)\end{array}$ \\
\hline $\begin{array}{l}\text { Direkt vor der Operation (vor ca. } \\
30^{\prime} 0,08 \mathrm{~g} \text { Morph. hydrochl. } \\
\text { subkutan) }\end{array}$ & 9 h $30^{\prime}$ vorm. & 0,090 \\
\hline $\begin{array}{l}\text { Sofort nach d. Operation } \\
30^{\prime} \text { " } \\
\text { (Blutentnahme) }\end{array}$ & $\begin{array}{l}\left.10^{\mathrm{h}} 20^{\prime} \text { vorm. (Operationsdauer } 50^{\prime}\right) \\
10^{\mathrm{H}} 20^{\prime}-50^{\prime} \text { vorm. }\left(30^{\prime}\right)\end{array}$ & $\begin{array}{l}0,128 \\
0,126\end{array}$ \\
\hline
\end{tabular}

Den Einfluss des Paukreashormons im obigen Hundeblut auf den Blutzucker eines Kaninchens sieht man im folgenden.

\begin{tabular}{|c|c|c|c|c|c|c|c|}
\hline \multirow[b]{2}{*}{$\begin{array}{c}\text { Menge d. } \\
\text { Blutes }\end{array}$} & \multirow[b]{2}{*}{$\begin{array}{l}\text { Methode d. } \\
\text { Isolierung } \\
\text { d. Hormons }\end{array}$} & \multirow[b]{2}{*}{$\begin{array}{l}\text { Menge d. } \\
\text { Extraktes }\end{array}$} & \multirow[b]{2}{*}{$\begin{array}{l}\text { Körp. } \\
\text { Gew. d. } \\
\text { Kanin. }\end{array}$} & \multicolumn{3}{|c|}{ Blutzucker $(\%)$} & \multirow{2}{*}{$\begin{array}{l}\text { Grad d. } \\
\text { Vermin- } \\
\text { derung } \\
\text { d. Blut- } \\
\text { zuckers }\end{array}$} \\
\hline & & & & $\begin{array}{l}\text { vor d. } \\
\text { Inj. }\end{array}$ & $\begin{array}{l}2 \frac{1}{2} \text { St. } \\
\text { nach d. } \\
\text { Inj. }\end{array}$ & $\begin{array}{l}4 \frac{1}{2} \text { St. } \\
\text { nach d. } \\
\text { Inj. }\end{array}$ & \\
\hline $140 \mathrm{cc}$ & $\begin{array}{c}\text { Alkohol- } \\
\mathrm{H}_{2} \mathrm{SO}_{4}\end{array}$ & $9 \mathrm{ccm}$ & $1400 \mathrm{~g}$ & 0,092 & 0,125 & 0,110 & $\begin{array}{l}\text { zugenom- } \\
\text { men }\end{array}$ \\
\hline
\end{tabular}

Fall 27.

Bei diesem Fall ist der Blutzucker während der Untersuchung unverändert geblieben, und das Alkoholextrakt des Hundeblutes konnte den Blutzucker des Kaninchens nur um 7\%, vermindern. 


\section{Tabelle XXII.}

(Fall 27)

Vers. Nr. 9. 27. V. 1925. Hund ô $14 \mathrm{~kg}$.

\begin{tabular}{c|l|c}
\hline & \multicolumn{1}{|c|}{ Zeit } & $\begin{array}{c}\text { Blutzucker } \\
(\%)\end{array}$ \\
\hline $\begin{array}{c}\text { Direkt vor der Operation (vor ca. } \\
30^{\prime} 0,14 \text { g Morph. hydrochl. }\end{array}$ & $9^{\text {h } 27^{\prime} \text { vorm. }}$ & 0,090 \\
subkutan) & & \\
Sofort nach d. Operation & $10^{\mathrm{h}} 15^{\prime}$ vorm. (Operationsdauer $\left.48^{\prime}\right)$ & 0,137 \\
$20^{\prime}$ & $10^{\mathrm{h}} 15^{\prime}-35^{\prime}$ vorm. $\left(20^{\prime}\right)$ & 0,137 \\
$45^{\prime}$ & $10^{\mathrm{h}} 35^{\prime}-11^{\mathrm{h}}$ vorm. $\left(25^{\prime}\right)$ & 0,135
\end{tabular}

Den Einfluss des Pankreashormons im obigen Hundeblute auf den Blutzucker eines Kaninchens findet man im folgenden.

\begin{tabular}{|c|c|c|c|c|c|c|c|}
\hline \multirow[b]{2}{*}{$\begin{array}{l}\text { Menge d. } \\
\text { Blutes }\end{array}$} & \multirow[b]{2}{*}{$\begin{array}{l}\text { Methode d. } \\
\text { Isolierung } \\
\text { d. Hormons }\end{array}$} & \multirow[b]{2}{*}{$\begin{array}{l}\text { Menge d. } \\
\text { Extraktes }\end{array}$} & \multirow{2}{*}{$\begin{array}{l}\text { Körp. } \\
\text { Gew. d. } \\
\text { Kanin. }\end{array}$} & \multicolumn{3}{|c|}{ Blutzucker (\%) } & \multirow{2}{*}{$\begin{array}{l}\text { Grad d. } \\
\text { Vermin- } \\
\text { derung } \\
\text { d. Blut- } \\
\text { zuckers }\end{array}$} \\
\hline & & & & $\begin{array}{l}\text { vor d. } \\
\text { Inj. }\end{array}$ & $\begin{array}{l}2 \frac{1}{2} \mathrm{St} . \\
\text { nach d. } \\
\text { Inj. }\end{array}$ & $\begin{array}{l}4 \frac{1}{2} \mathrm{St} . \\
\text { nach d. } \\
\text { Inj. }\end{array}$ & \\
\hline $140 \mathrm{co}$ & $\begin{array}{c}\text { Alkohol- } \\
\mathrm{H}_{2} \mathrm{SO}_{4}\end{array}$ & $12 c$ & $1380 \mathrm{~g}$ & 0,086 & 0,080 & 0,089 & $7 \%$ \\
\hline
\end{tabular}

III. Pankreashormon im Hundeblut, das vor und nach der Vagusreizung einem Hunde entnommen wurde.

Bei den zu dieser Gruppe gehörigen Füllen war die Behandlung dieselbe wie bei den anderen, aber bei ihnen wurden ca. $30 \mathrm{ccm}$ Blut durch die Carotiskanüle vor der Vagusreizung entnommen; endlich liessen wir sie sich nach der Vagusreizung bis zum Tod verbluten. Aus dem Blut präparierten wir Pankreashormon nach der schon beschriebenen Alkohol-Schwefelsäure-Methode oder A ceton-Pikrinsäure-Methode und bestimmten die durch. Injektion dieses Pankreashormons hervorgerufene Blutzuckerverminderung des Kaninchens.

\section{Fall 28.}

Bei diesem Fall ist der Blutzucker, wie in Tabelle XXIII gezeigt wird, durch Vagusreizung unverändert geblieben. Das mittels der Aceton-Pikrinsäurc-Methode bereitete Pankreashormon aus $30 \mathrm{ccm}$ Blut, welches vor der Vagusreizung entnommen wurde, setzte den Blutzucker des Kaninchens 
bei der Injektion um 5\% herab. Dasjenige aber aus $30 \mathrm{ccm}$ Blut, welches nach der Vagusreizung entnommen wurde, verminderte den Blutzucker des Kaninchens um 15\%. Aber das Alkoholextrakt, das aus $30 \mathrm{ccm}$ des zuletzt entnommenen Hundeblutes dargestellt wurde, hatte keine Kraft, den Blutzucker des Kaninchens zu vermindern.

\section{Tabelle XXIII.}

(Fall 28)

Vers Nr. 10. 5. VI. 1925. Hund ô $8,8 \mathrm{~kg}$.

\begin{tabular}{l|l|c}
\hline & \multicolumn{1}{|c|}{ Zeit } & $\begin{array}{c}\text { Blutzucker } \\
(\%)\end{array}$ \\
\hline $\begin{array}{c}\text { Direkt vor der Operation (vor ca. } \\
30^{\prime} 0,09 \mathrm{~g} \text { Morph. hydrochl. } \\
\text { subkutan) }\end{array}$ & $9^{\mathrm{h}} \overline{5}^{\prime}$ vorm. & 0,099 \\
$\begin{array}{c}\text { Sofort nach d. Operation (Blut- } \\
\text { entnahme 30 ccm) } \\
\text { Reizungsperiode (Blutentnahme) }\end{array}$ & $10^{\mathrm{h}}$ vorm. (Operationsdauer $\left.55^{\prime}\right)$ & 0,110 \\
$10^{\mathrm{h}}-10^{\mathrm{h}} 10^{\prime}$ vorm. $\left(10^{\prime}\right)$ & 0,113
\end{tabular}

Der Einfluss des Pankreashormons im obigen Hundeblute auf den Blutzucker eines Kaninchens ist wie folgt.

\begin{tabular}{|c|c|c|c|c|c|c|c|}
\hline \multirow{2}{*}{$\begin{array}{c}\text { Zeit d. } \\
\text { Blutent- } \\
\text { nahme }\end{array}$} & \multirow[b]{2}{*}{$\begin{array}{c}\text { Menge d. } \\
\text { Blutes }\end{array}$} & \multirow[b]{2}{*}{$\begin{array}{l}\text { Methode d. } \\
\text { Isolierung } \\
\text { d. Hormons }\end{array}$} & \multirow{2}{*}{$\begin{array}{l}\text { Körp. } \\
\text { Gew. d. } \\
\text { Kanin. }\end{array}$} & \multicolumn{3}{|c|}{ Blutzucker (\%) } & \multirow{2}{*}{$\begin{array}{l}\text { Grad d. } \\
\text { Vermin- } \\
\text { derung } \\
\text { d. Blut- } \\
\text { zuckers }\end{array}$} \\
\hline & & & & $\begin{array}{l}\text { ror d. } \\
\text { Inj. }\end{array}$ & $\begin{array}{l}2 \frac{1}{2} \text { St. } \\
\text { nach d. } \\
\text { Inj. }\end{array}$ & $\begin{array}{l}4 \frac{1}{2} \mathrm{St} . \\
\text { nach d. } \\
\text { Inj. }\end{array}$ & \\
\hline $\begin{array}{l}\text { vor d. } \\
\text { Reiz. }\end{array}$ & $30 \mathrm{ccm}$ & Acet.-Pikr. & $1150 \mathrm{~g}$ & 0,088 & 0,084 & 0,087 & $5 \%$ \\
\hline nach d. & $"$ & $"$ & 1120, & 0,098 & 0,084 & 0,091 & $15 \%$ \\
\hline Reiz. & $"$ & $\underset{\mathrm{H}_{2} \mathrm{SO}_{4}}{\text { Alkohol- }}$ & $1240 "$ & 0,089 & 0,090 & 0,095 & $\begin{array}{l}\text { fast un- } \\
\text { verändert }\end{array}$ \\
\hline
\end{tabular}

Fall 29.

Bei diesem Falle nahm Blutzucker durch Vagusreizung deutlich ab ; das Pankreashormon aus dem nach der Vagusreizung entnommenen Hundeblut, das durch die Aceton-Pikrinsüure-Methode bereitet wurde, zeigte dentlichen Einfluss auf den Blutzucker des Kaninchens, nämlich 12\% ige Verminderung, während dasjenige aus dem Blut vor der Vagusreizung keine Verminderung hervorrief. Aber das Alkoholextrakt, welches aus dem Hundeblut nach der Vagusreizung dargestellt wurde, liess Kaninchenblutzucker durch Injektion nicht sinken. Wir können das in Tabelle XXIV im Detail sehen. 
Fall 30.

Wie man aus nachfolgender Tabelle ersieht, nahm der Blutzucker bei diesem Falle durch Vagusreizung nicht deutlich ab. Wenn man den Einfluss des Pankreashormons im Hundeblut, das durch die Aceton-Pikrinsäure-Methode präpariert wurde, auf den Kaninchenblutzucker betrachtet, findet man, dass er anfangs wirkungslos war, aber durch V agusreizung deutliche, d. h. $15 \%$ blutzuckervermindernde $\mathrm{Kraft}$ gewonnen hat. Aber das Alkoholextrakt aus letzterem Blut zeigte keinen Einfluss auf Kaninchenblutzucker.

\section{Tabelle XXIV.}

(Fall 29)

Vers. Nr. 11. 10. VI. 1925. Hund $q 11 \mathrm{~kg}$.

\begin{tabular}{|c|c|c|}
\hline & Zeit & $\begin{array}{c}\text { Blutzucker } \\
(\%)\end{array}$ \\
\hline $\begin{array}{l}\text { Direkt vor der Operation (vor ca. } \\
30^{\prime} 0,11 \mathrm{~g} \text { Morph. hydrochl. }\end{array}$ & $10^{\mathrm{h} 3 /}$ vorm. & 0,159 \\
\hline $\begin{array}{l}\text { Sofort nach der Operation (Blut- } \\
\text { entnahme } 30 \mathrm{ccm} \text { ) }\end{array}$ & $10^{\mathrm{b}} 50^{\prime}$ vorm. (Operationsdauer $47^{\prime}$ ) & 0,180 \\
\hline Reizungsperiode (Blutentnahme) & $10^{\mathrm{b}} 50^{\prime}-11^{\mathrm{h}} 10^{\prime}$ vorm. $\left(20^{\prime}\right)$ & 0,136 \\
\hline
\end{tabular}

Der Einfluss des Pankreashormons im obigen Hundeblute auf den Blutzucker eines Kaninchens ist wie folgt.

\begin{tabular}{|c|c|c|c|c|c|c|c|}
\hline \multirow[b]{2}{*}{$\begin{array}{l}\text { Zeit d. } \\
\text { Blutent- } \\
\text { nahme }\end{array}$} & \multirow[b]{2}{*}{$\begin{array}{l}\text { Menge d. } \\
\text { Blutes }\end{array}$} & \multirow{2}{*}{$\begin{array}{l}\text { Methode d. } \\
\text { Isolierung } \\
\text { d. Hormons }\end{array}$} & \multirow[b]{2}{*}{$\begin{array}{l}\text { Körp. } \\
\text { Gew. d. } \\
\text { Kanin. }\end{array}$} & \multicolumn{3}{|c|}{ Blutzucker $(\%)$} & \multirow{2}{*}{$\begin{array}{l}\text { Grad d. } \\
\text { Vermin- } \\
\text { derung } \\
\text { d. Blut- } \\
\text { zuckers }\end{array}$} \\
\hline & & & & $\begin{array}{l}\text { vor d. } \\
\text { Inj. }\end{array}$ & $\begin{array}{l}2 \frac{1}{2} S t . \\
\text { nach d. } \\
\text { Inj. }\end{array}$ & $\begin{array}{l}4 \frac{1}{2} \mathrm{St} . \\
\text { nach d. } \\
\text { Inj. }\end{array}$ & \\
\hline $\begin{array}{l}\text { vor d. } \\
\text { Reiz. }\end{array}$ & $30 \mathrm{ccm}$ & Acet.-Pikr. & $1320 \mathrm{~g}$ & 0,083 & 0,084 & 0,092 & $\begin{array}{l}\text { fast un- } \\
\text { verändert }\end{array}$ \\
\hline \multirow{2}{*}{$\begin{array}{c}\text { nach d. } \\
\text { Reiz. }\end{array}$} & $"$ & & $1220 "$ & 0,087 & 0,076 & 0,089 & $12 \%$ \\
\hline & " & $\begin{array}{c}\text { Alkohol- } \\
\mathrm{H}_{z} \mathrm{SO}_{4}\end{array}$ & 1270, & 0,094 & 0,096 & 0,010 & $\begin{array}{c}\text { fast un- } \\
\text { verändert }\end{array}$ \\
\hline
\end{tabular}

Tabelle XXV.

(Fall 30)

Vers. Nr. 12. 6. VI. 1925. Hund $\delta 8 \mathrm{~kg}$.

\begin{tabular}{|c|c|c|}
\hline & Zeit & $\begin{array}{c}\text { Blutzucker } \\
(\%)\end{array}$ \\
\hline $\begin{array}{l}\text { Direkt vor der Operation (vor ca. } \\
30 \prime 0,08 \mathrm{~g} \text { Morph. hydrochl. } \\
\text { subkutan) } \\
\text { Sofort nach der Operation (Blut- } \\
\text { entnahme } 30 \mathrm{ccm} \text { ) } \\
\text { Reizungsperiode (Blutentnahme) }\end{array}$ & $\begin{array}{l}9^{\mathrm{h}} 10^{\prime} \text { vorm. } \\
10^{\mathrm{h}} \text { vorm. (Operationsdauer } 50^{\prime} \text { ) } \\
10^{\mathrm{h}}-10^{\mathrm{h}} 10^{\prime} \text { vorm. }\left(10^{\prime}\right)\end{array}$ & $\begin{array}{l}0,141 \\
0,149 \\
0,137\end{array}$ \\
\hline
\end{tabular}


Den Einfluss des Pankreashormons im obigen Hundeblute auf den Blutzucker eines Kaninchens sieht man im folgenden.

\begin{tabular}{|c|c|c|c|c|c|c|c|}
\hline \multirow{2}{*}{$\begin{array}{l}\text { Zeit d. } \\
\text { Blutent- } \\
\text { nahwe }\end{array}$} & \multirow[b]{2}{*}{$\begin{array}{c}\text { Menge d. } \\
\text { Blutes }\end{array}$} & \multirow{2}{*}{$\begin{array}{l}\text { Methode d. } \\
\text { Isolierung } \\
\text { d. Hormons }\end{array}$} & \multirow{2}{*}{$\begin{array}{l}\text { Körp. } \\
\text { Gew.d. } \\
\text { Kanin. }\end{array}$} & \multicolumn{3}{|c|}{ Blutzucker $(\%)$} & \multirow{2}{*}{$\begin{array}{c}\text { Grad d. } \\
\text { Vermin- } \\
\text { derung } \\
\text { d. Blut- } \\
\text { zuckers }\end{array}$} \\
\hline & & & & $\begin{array}{l}\text { vor d. } \\
\text { Inj.j. }\end{array}$ & $\begin{array}{l}2 \frac{1}{2} \text { St. } \\
\text { nach d. } \\
\text { Inj. }\end{array}$ & $\begin{array}{l}4 \frac{1}{2} \text { St. } \\
\text { nach d. } \\
\text { Inj. }\end{array}$ & \\
\hline $\begin{array}{l}\text { vor d. } \\
\text { Reiz. }\end{array}$ & $30 \mathrm{ccm}$ & Acet.-Pikr. & $1100 \mathrm{~g}$ & 0,080 & 0,081 & 0,084 & $\begin{array}{l}\text { fast un- } \\
\text { veründert }\end{array}$ \\
\hline \multirow{2}{*}{$\begin{array}{l}\text { nach d. } \\
\text { Reiz. }\end{array}$} & $"$ & & $1200 "$ & 0,088 & 0,076 & 0,081 & $15 \%$ \\
\hline & $"$ & $\begin{array}{c}\text { Alkohol- } \\
\mathrm{H}_{2} \mathrm{SO}_{4}\end{array}$ & $1300 "$ & 0,078 & 0,074 & 0,080 & $\begin{array}{l}\text { fast un- } \\
\text { verïndert }\end{array}$ \\
\hline
\end{tabular}

Ich fasse hier alle obigen Versuche bei Hunden übersichtshalber in den folgenden 3 Tabellen zusammen :

\section{Tabelle XXVI.}

Pankreashormon im Blute der Hunde, bei welchen N. vagus gereizt wurde.

\begin{tabular}{|c|c|c|c|c|c|c|c|c|c|}
\hline \multirow[b]{2}{*}{$\begin{array}{l}\text { Vers. Nr. } \\
\text { u. Datum }\end{array}$} & \multirow[b]{2}{*}{ 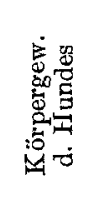 } & \multirow{2}{*}{ 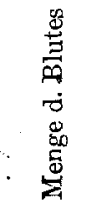 } & \multirow[b]{2}{*}{ 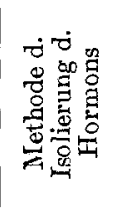 } & \multirow{2}{*}{ 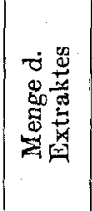 } & \multirow[b]{2}{*}{ 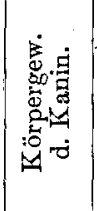 } & \multicolumn{3}{|c|}{ Blutzucker } & \multirow[b]{2}{*}{ 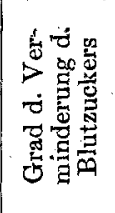 } \\
\hline & & & & & & $\begin{array}{l}\dot{\vec{g}} \\
\dot{0} \\
\dot{\vec{b}}\end{array}$ & 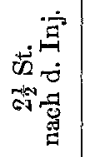 & 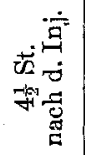 & \\
\hline 9. V. 1925 & $9,8 \mathrm{~kg}$ & $140 \mathrm{ccm}$ & $\begin{array}{c}\text { Alknhol- } \\
\mathrm{H}_{2} \mathrm{SO}_{4} \\
\text { Methode }\end{array}$ & $10 \mathrm{ccm}$ & $1280 \mathrm{~g}$ & 0,104 & 0,055 & 0,071 & $47 \%$ \\
\hline 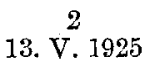 & 18,8, & $"$ & $"$ & 9 , & 1470, & 0,102 & 0,090 & 0,071 & 30 \\
\hline 20. V. 1.925 & 6,5, & $n$ & $"$ & 11, & 1500, & 0,092 & 0,069 & 0,090 & 26, \\
\hline 6. V. 1925 & 8,5, & $"$ & $"$ & 10, & $\cdot 1600 "$ & 0,090 & 0,061 & 0,078 & 32, \\
\hline 25. V. 1925 & 8,5, & $n$ & $"$ & $9 "$ & 1400, & 0,089 & 0,089 & 0,104 & $\begin{array}{l}\text { fast un- } \\
\text { veründert }\end{array}$ \\
\hline
\end{tabular}

Aus der Tabelle ersieht man deutlich, dass das Extrakt aus Blut der Hunde, bei welchen $N$. vagus gereizt wurde, ziemlich stark auf den Kaninchenblutzucker vermindernd wirkt. Der Fall 8 bildete eine Ausnahme : sein Extrakt war gar nicht wirksam. 
Tabelle XXVII.

Pankreashormon im Blute der Hunde, bei welchen zur Kontrolle $\mathrm{N}$. vagus nicht gereizt wurde

\begin{tabular}{|c|c|c|c|c|c|c|c|c|c|}
\hline \multirow[b]{2}{*}{$\begin{array}{l}\text { Vers. Nr. } \\
\text { u. Datum }\end{array}$} & \multirow[b]{2}{*}{ 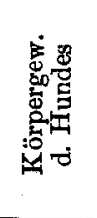 } & \multirow{2}{*}{ 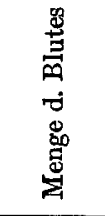 } & \multirow[b]{2}{*}{ 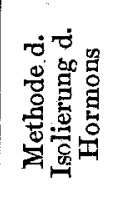 } & \multirow[b]{2}{*}{ 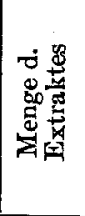 } & \multirow[b]{2}{*}{ 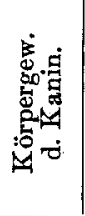 } & \multicolumn{3}{|c|}{ Blutzucker (\%) } & \multirow[b]{2}{*}{ 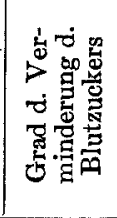 } \\
\hline & & & & & & $\begin{array}{l}\text { 完 } \\
\dot{0} \\
\dot{0} \\
\dot{0}\end{array}$ & 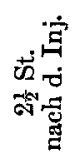 & 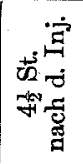 & \\
\hline 16. V. 1925 & $10,5 \mathrm{~kg}$ & $140 \mathrm{ccm}$ & $\begin{array}{c}\text { Alkohol- } \\
\mathrm{H}_{2} \mathrm{SO}_{4} \\
\text { Methode }\end{array}$ & $12 \mathrm{ccm}$ & $1320 \mathrm{~g}$ & 0,090 & 0,084 & 0,087 & $7 \%$ \\
\hline $22 . \stackrel{5}{V^{5}} 1925$ & $17,0 "$ & $"$ & 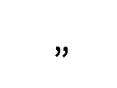 & $"$ & 1200, & 0,085 & 0,081 & 0,088 & 6, \\
\hline $23, \mathrm{~V}^{6} .1925$ & 7,5, & $"$ & $"$ & $"$ & 1400, & 0,092 & 0,125 & 0,116 & $\begin{array}{c}\text { zuge- } \\
\text { nommen }\end{array}$ \\
\hline 27. ${ }^{9} .1925$ & 14,0 & $"$ & $"$ & $"$ & 1380, & 0,086 & 0,080 & 0,089 & $7 \%$ \\
\hline
\end{tabular}

Tabelle XXVIII.

Pankreashormon im Blute desselben Hundes, das ihm zweizeitig vor und nach Vagusreizung entnommen wurde.

\begin{tabular}{|c|c|c|c|c|c|c|c|c|c|}
\hline \multirow[b]{2}{*}{ 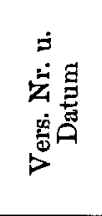 } & \multirow[b]{2}{*}{ 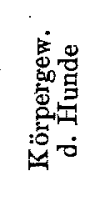 } & \multirow[b]{2}{*}{ 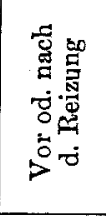 } & \multirow{2}{*}{ 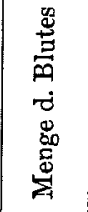 } & \multirow[b]{2}{*}{ 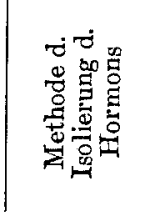 } & \multirow[b]{2}{*}{ 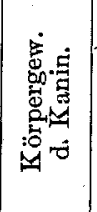 } & \multicolumn{3}{|c|}{ Blutzucker $(\%)$} & \multirow[b]{2}{*}{ 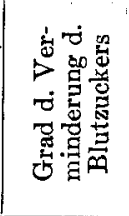 } \\
\hline & & & & & & $\begin{array}{l}\dot{\vec{\Xi}} \\
\dot{\vec{D}} \\
\dot{0}\end{array}$ & 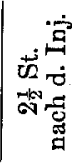 & 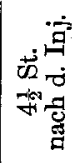 & \\
\hline $\begin{array}{l}10 \\
\text { 5. VI. } \\
1925\end{array}$ & $8,8 \mathrm{~kg}$ & $\begin{array}{l}\text { vor d. } \\
\text { Reizung } \\
\text { nach d. } \\
\text { Reizung }\end{array}$ & $30 \mathrm{ccm}$ & $\begin{array}{c}\text { Aceton- } \\
\text { Pikrinsiure- } \\
\text { Methode } \\
"\end{array}$ & $\begin{array}{l}1150 \mathrm{~g} \\
1120 "\end{array}$ & $\begin{array}{l}0,088 \\
0,098\end{array}$ & $\begin{array}{l}0,084 \\
0,084\end{array}$ & $\begin{array}{l}0,087 \\
0,091\end{array}$ & $\begin{array}{l}5 \% \\
15 \%\end{array}$ \\
\hline $\begin{array}{c}11 \\
10 . \mathrm{VI} . \\
1925\end{array}$ & 11,0, & $\begin{array}{c}\text { vor d. } \\
\text { Reizung } \\
\\
\text { nach d. } \\
\text { Reizung }\end{array}$ & $"$ & $"$ & $\begin{array}{l}1320, \\
1220,\end{array}$ & $\begin{array}{l}0,084 \\
0,087\end{array}$ & $\begin{array}{l}0,084 \\
0,076\end{array}$ & $\begin{array}{l}0,092 \\
0,089\end{array}$ & $\begin{array}{c}\text { fast un- } \\
\text { veründert } \\
12 \%\end{array}$ \\
\hline $\begin{array}{c}12 \\
10 . \mathrm{VI} . \\
1925\end{array}$ & 8,0, & $\begin{array}{l}\text { vor d. } \\
\text { Reizung } \\
\text { nach d. } \\
\text { Reizung }\end{array}$ & $"$ & $"$ & $\begin{array}{l}1100, " \\
1200, "\end{array}$ & $\begin{array}{l}0,080 \\
0,088\end{array}$ & $\begin{array}{l}0,081 \\
0,076\end{array}$ & $\begin{array}{l}0,084 \\
0,081\end{array}$ & $\begin{array}{c}\text { fast un- } \\
\text { verändert } \\
15 \%\end{array}$ \\
\hline
\end{tabular}

Wie aus diesen Tabellen leicht ersichtlich, nahm der Blutzucker bei Hunden durch Vagusreizung zumeist ab, obgleich er bei den Kontrollver- 
suchen fast unverändert geblieben ist ; diese Resultate stimmen mit denen im Kapitel I überein.

Das Pankreashormon im Blute des Hundes nahm durch Vagusreizung im allgemeinen auffallend zu : das Alkoholextrakt, das aus $140 \mathrm{ccm}$ des nach Vagusreizung entnommenen Blutes extrahiert wurde, verminderte Kanichenblutzucker, ausser einem unveränderten Fall, un 26 bis $47 \%$, im Durchschnitt ca. $36 \%$, bei den Kontrollfällen, auch ausser einem unveränderten Fall, um 6 bis $7 \%$, im Durchschnitt um ca. $7 \%$. Das Pankreashormon in $30 \mathrm{ccm}$ Hundeblut, das vor Vagusreizung von einem Hunde entnommen und durch die Aceton-Pikrinsüure-Methode dargestellt worden war, verminderte Kaninchenblutzucker bei einem Fall um $5 \%$, bei 2 Fällen gar nicht, während das aus dem nach Vagusreizung entnommenen wirksamer war, so dass es den Kaninchenblutzucker um 12 bis $15 \%$ herabsetzte.

\section{B. Versuche bei Kaninchen.}

I. Pankreashormon im Blute von Kaninchen, bei welchen N. vagus gereizt wurde.

Im vorigen Kapitel wurde die Technik eingehend geschildert, deshalb will ich hier nur kurz darauf eingehen. Bei 24 Stunden lang hungernden Kaninchen wurden beiderseitige Nn. vagi mit oder ohne Narkose von Urethan oder Äther am Halse durchschnitten und in der Bauchhöhle von dem unteren Oesophagusteil abpräpariert und in die schon erwähnte besondere Elektrode gesteckt und gereizt.

Der Blutzucker der Kaninchen stieg im allgemeinen während der Untersuchung immer mehr und zeigte keine deutliche Beeinflussung der Vagusreizung. Diese Ergebnisse stimmen mit denen im vorigen Kapitel überein. Obgleich die Pankreashormonsekretion sich vermehrt, kann doch das Hormon Blutzucker nicht deutlich vermindern, weil das Adrenalsystem des Kaninchens wahrscheinlich zu empfindlich ist und dadurch hervorgerufene Glykolyse lebhaft stattfindet. Folglich muss man das Pankreashormon im Blute des Kaninchens direkt bestimmen, um den wahren Einfluss der Vagusreizung auf die innere Sekretion des Pankreas zu finden. Nach Beendigung der Reizung des Vagus wurde das Blut mittelst der Carotiskanüle bis zum Tode des Tieres entnommen ; das Pankreashormon im Blute wurde durch die schon oft genannte Alkohol-Schwefelsäure-Methode oder AcetonPikrinsäure-Methode präpariert und unter die Haut der Kaninchen gespritzt und der Blutzucker bei ihnen vor und nach der Injektion bestimmt. 
Die Versuche (Fall 31-38) seien in folgender Tabelle zusammengefasst :

Tabelle XXIX.

(Fall 31-38)

Pankreashormon im Blute der Kaninchen, bei welchen

$\mathrm{N}$. vagus gereizt wurde.

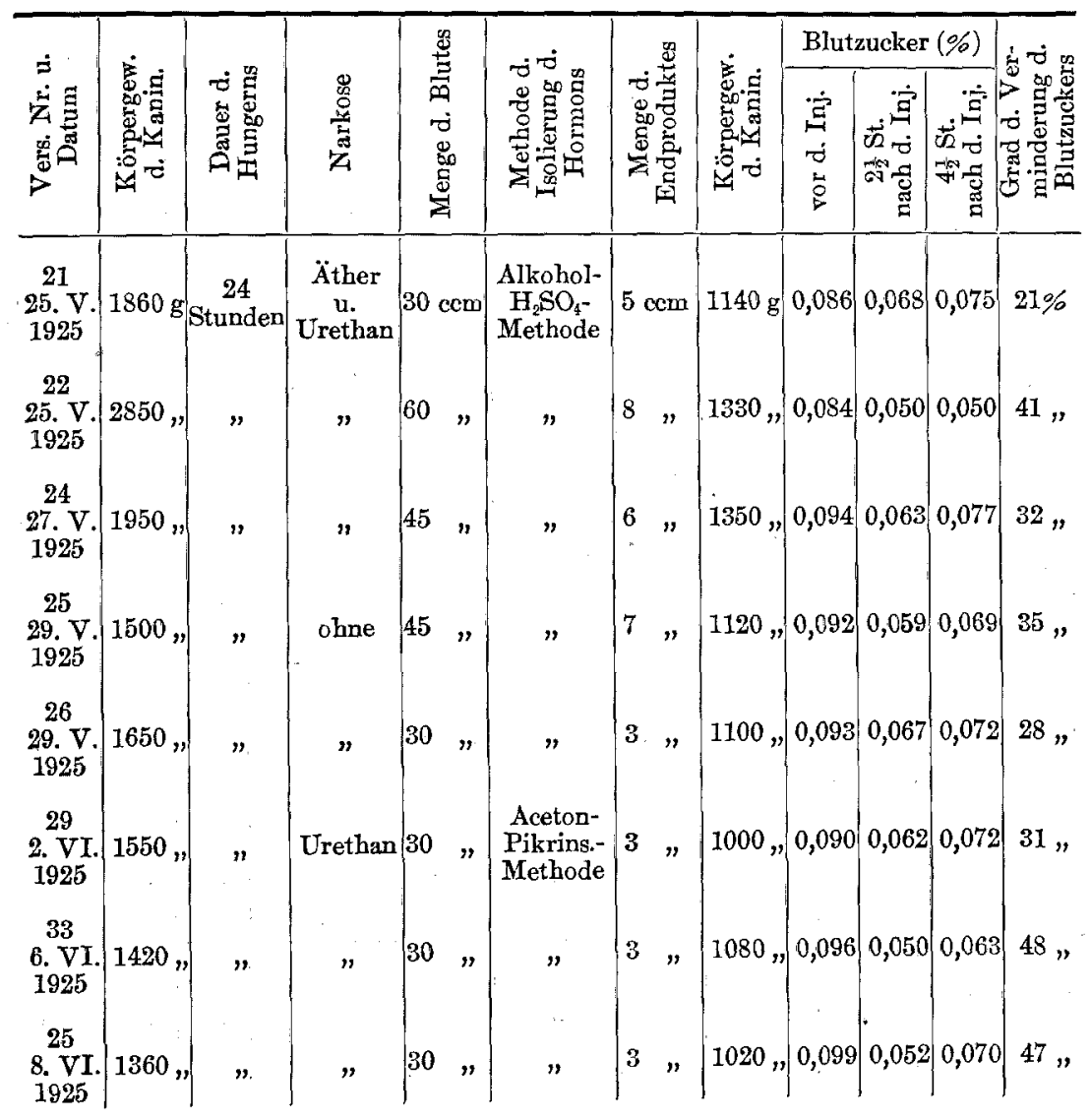

Wie aus obiger Tabelle ersichtlich verminderte das Pankreashormon in $30 \mathrm{ccm}$ Blut, das durch die Aceton-Pikrinsäure-Methode präpariert wurde, Kaninchenblutzucker, ausser bei einem Fall, um 31 bis $48 \%$, im Durchschnitt um 42\%. Auch sein Alkoholextrakt liess Kaninchenblutzucker auffallend sinken. 
II. Pankreashormon im Blute der Kaninchen, bei welchen der Vagus zur Kontrolle nicht gereizt wurde.

Bei diesen Fällen (Fall 39-42) wurde der operative Eingriff ebenso wie bei den vorigen ausser elektrischer Reizung ausgeführt. Die Resultate werden in folgender Tabelle zusammengefasst:

Tabelle XXX.

(Fall 39-42)

Pankreashormon im Blute der Kaninchen, bei welchen zur Kontrolle N. vagus nicht gereizt wurde.

\begin{tabular}{|c|c|c|c|c|c|c|c|c|c|c|c|}
\hline \multirow[b]{2}{*}{ 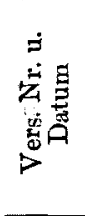 } & \multirow[b]{2}{*}{ 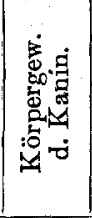 } & \multirow[b]{2}{*}{ 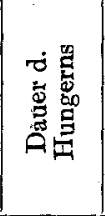 } & \multirow[b]{2}{*}{ 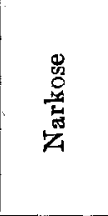 } & \multirow{2}{*}{ 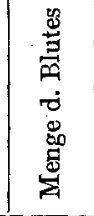 } & \multirow[b]{2}{*}{ 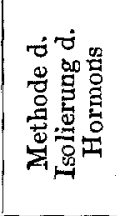 } & \multirow{2}{*}{ 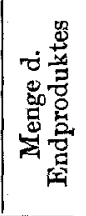 } & \multirow[b]{2}{*}{ 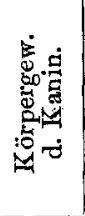 } & \multicolumn{3}{|c|}{ Blutzucker $(\%)$} & \multirow[b]{2}{*}{ 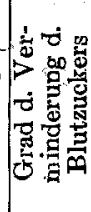 } \\
\hline & & & & & & & & $\begin{array}{l}\vec{E} \\
\vec{E} \\
\dot{\vec{E}} \\
\dot{\vec{b}}\end{array}$ & 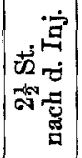 & 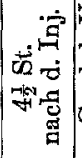 & \\
\hline $\begin{array}{l}28 \\
2 . \mathrm{VI} . \\
1925\end{array}$ & $1340 \mathrm{~g}$ & Stunden & Urethan & $45 \mathrm{ccm}$ & $\begin{array}{l}\text { Aceton- } \\
\text { Pikrins.- } \\
\text { Methode }\end{array}$ & $|4,5 \mathrm{ccm}|$ & $1180 \mathrm{~g}$ & 0,100 & 0,074 & 0,079 & $26 \%$ \\
\hline $\begin{array}{c}30+31 \\
3 . \mathrm{VI} . \\
1925\end{array}$ & $\begin{array}{l}1150, \\
1350 "\end{array}$ & " & ohne & $30 "$ & $"$ & $3,0 \quad$, & $1030, n$ & 0,080 & 0,068 & $0,073 \mid$ & $16 n$ \\
\hline $\begin{array}{c}36+37 \\
3 . \mathrm{VI} . \\
1925\end{array}$ & $\begin{array}{l}1250, \\
1080,\end{array}$ & $"$ & $"$ & $60 \%$ & " & 6,0 & 1290, & 0,097 & 0,068 & 0,080 & 30 \\
\hline $\begin{array}{l}32 \\
4 . V I \\
1925\end{array}$ & 2800, & $\eta$ & Äther & $20 "$ & $n$ & 2,0, & $1100 "$ & 0,091 & 0,079 & 0,085 & 14 \\
\hline
\end{tabular}

Aus obiger 'Tabelle ist ersichtlich, dass das Pankreashormon im Blute der Kontrollfälle viel geringer als bei den vorigen ist und zwar ungefähr um die Hälfte. Ferner scheint es mir, dass es keinen Unterschied der Pankreashormonmenge im Blute zwischen den mit $\ddot{A}$ ther oder Urethan narkotisierten Kaninchen und den nicht narkotisierten gibt. 


\section{Pankreashormon im Bluteder Kaninchen, bei welchen beiderseitige Nn. splanchnici durchschnitten wurden.}

Bei diesen Fällen (Fall 43-50) war die Untersuchungstechnik ebenso wie bei den anderen; aber beiderseitige Nn. splanchnici wurden oberhalb des Ganglion coeliacum vor der Vagusreizung durchschnitten. N. sympathicus wurde dadurch freilich von den Nebennieren und auch von dem Pankreas abgetrennt. Die Versuchsresultate bei den splanchnikotomierten Kaninchen werden in folgender Tabelle zusammengefasst.

\section{Tabelle XXXI. \\ (Fall 43-50)}

Pankreashormon im Blute der Kaninchen, bei welchen

N. splanchnicus durchschnitten wurde.

\begin{tabular}{|c|c|c|c|c|c|c|c|c|c|}
\hline \multirow[b]{2}{*}{ 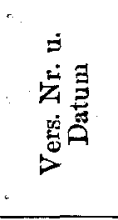 } & \multirow[b]{2}{*}{ 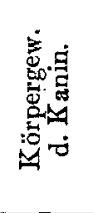 } & \multirow[b]{2}{*}{ 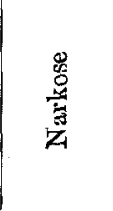 } & \multirow[b]{2}{*}{ 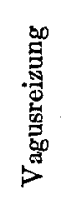 } & \multirow{2}{*}{ 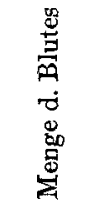 } & \multirow[b]{2}{*}{ 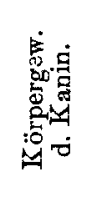 } & \multicolumn{3}{|c|}{ Blutzucker (\%) } & \multirow[b]{2}{*}{ 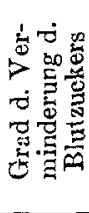 } \\
\hline & & & & & & $\begin{array}{l}\dot{\vec{E}} \\
\dot{0} \\
\dot{0} \\
\dot{0}\end{array}$ & 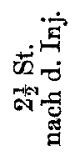 & 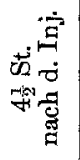 & \\
\hline 24. $\stackrel{44}{\mathrm{VI} .}$ & $1450 \mathrm{~g}$ & Ather & $(-)$ & $30 \mathrm{ccm}$ & $1310 \mathrm{~g}$ & 0,094 & 0,077 & 0,083 & $17 \%$ \\
\hline $\begin{array}{c}45 \\
24 . \mathrm{VI} . \\
1925\end{array}$ & $1560 "$ & $n$ & $(-)$ & $30 "$ & 1450, & 0,099 & 0,080 & 0,090 & 20, \\
\hline 10.38 & 1500 & Urethan & $(+)$ & 10) & & & & & \\
\hline $\begin{array}{c}1925 \\
39\end{array}$ & & & & & 1200, & 0,093 & 0,065 & 0,073 & 31, \\
\hline $\begin{array}{c}\text { 10. VI. } \\
1925 \\
40\end{array}$ & $1900 "$ & $"$ & $(+)$ & $20)$ & 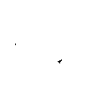 & & & & \\
\hline $\begin{array}{c}\text { 19. VI. } \\
1925\end{array}$ & $1200 n$ & $"$ & $(+)$ & $30 "$ & $1250 "$ & 0,096 & 0,069 & 0,081 & 28, \\
\hline $\begin{array}{c}42 \\
20 . \mathrm{VI} \\
1925\end{array}$ & 1350 & Äther & $(+)$ & $30 "$ & 1200 & 0,086 & 0,064 & 0,076 & $27 "$ \\
\hline $\begin{array}{l}43 \\
20 . \mathrm{VI} \\
1925\end{array}$ & 1400 & $"$ & $(+)$ & $20 "$ & 1500, & 0,097 & 0,076 & 0,078 & 30, \\
\hline $\begin{array}{l}\text { 24. }{ }^{46} \mathrm{VI} . \\
1925\end{array}$ & 1550, & $"$ & $(t)$ & 30, & 1080 & 0,100 & 0,070 & 0,082 & $40 "$ \\
\hline
\end{tabular}

Alle Kaninchen wurden 24 Stunden lang gehungert.

Pankreashormon wurde bei allen Füllen durch Aceton-Pikrinsïure-Methode isoliert.

Je ron $10 \mathrm{ccm}$ Blut gewonnenes Pankreashormon wurde in je $1 \mathrm{ccm}$ anges̈̈uerten Wassers gelöst. 
Wie aus obiger Tabelle ersichtlich, ist die blutzuckervermindernde Wirkung des Pankreashormons in $30 \mathrm{ccm}$ Blut, das vagusgereizten Tieren entnommen wurde, viel grösser als die aus nicht gereizten, d. h. erstere konnte Kaninchenblutzucker um 27 bis $40 \%$, im Durchschnitt um 32\%, aber letztere nur um 17 und 20\%, im Durchschnitt um 19\% herabsetzen. Vergleicht man den Hormongehalt des Blutes des splanchnikotomierten und nicht vagusgereizten Kaninchens mit dem des normalen Kaninchenblutes der Tabelle XXX, so findet man keinen Unterschied zwischen den beiden. Also sieht man, dass die Splanchnikotomie allein keinen Einfluss auf die innere Sekretion des Pankreas ausübt.

Wenn wir alle obigen Versuche bei den Kaninchen zusammenfassen, so geht daraus Folgendes hervor :

1. Der Blutzucker der Kaninchen vermehrte sich immer während der Untersuchung unabhängig von der Narkose und der Vagusreizung.

2. Im Blute der vagusgereizten $\mathrm{Kaninchen}$ ist bedeutend mehr Pankreashormon enthalten als in dem der nicht gereizten, und es ist auch unabhängig von der Splanchnikotomie und der Narkose.

Nun ist es nach Tscheboksaroff, ${ }^{11}$ Asher, ${ }^{12}$ Biedl, ${ }^{132}$ Elliot ${ }^{14)}$ u. a. sicher aufgeklärt, dass die Adrenalinsekretion des Nebennierenmarks durch N. splanchnicus befördert wird. Ich ${ }^{15}$ habe auch schon die Innervation der Nebenniere studiert und den $\mathrm{N}$. splanchnicus als den sekretorischen Nerven nachgewiesen. Ich habe N. splanchnicus oder N. vagus bei den Kaninchen durchschnitten und die dadurch hervorgerufene Nervenveränderung mikroskopisch verfolgt und schliesslich N. splanchnicus und zwar nur ihn allein als den Nerven des Nebennierenmarks nachgewiesen. Tsch e boks a r of $\mathrm{f}$ äusserrte sich auch dahin, dass N. vagus keinen Einfluss auf die Adrenalinsekretion ausübt. Aus den verschiedenen obigen Forschungen ergibt sich Folgendes: Der adrenalinsekretorische Nerv ist $\mathbf{N}$. splanchnicus und wahrscheinlich nur er allein. Hier ist es auch von mir sicher nachgewiesen, dass die innere Sekretion des Pankreas vom N. vagus beherrscht wird. Ich glaube auch, dass sie durch N. vagus allein innerviert wird, weil die Menge des Pánkreashormons im Blute unabhängig von der Splanchnikotomie ist. Wenn es im N. splanchnicus Hemmungsfasern für die Sekretion des Pankreashormons gübe, so müsste das Pankreashormon im Blute durch Splanchnikotomie mehr oder weniger zunehmen, was aber in der Tat nicht der Fall ist. Wenn es umgekehrt Beförderungsfasern für die Pankreashormonsekretion im N. splanchnicus gäbe, so müsste das Pankreashormon im Blute mehr oder weniger durch Splanchnikotomie abnehmen; ferner können wir uns nicht denken, dass die Funktion eines Organs 
in doppelter Weise durch Sympathicus und Parasympathicus gefördert wird. Aus allen obigen Versuchen bei Kaninchen fanden wir, dass $\mathrm{N}$. vagus und wahrscheinlich er allein die innere Sekretion des Pankreas beherrscht. Wenn wir endlich die Innervation der Innensekretion des Pankreas mit der der Nebenniere vergleichen, so finden wir, dass erstere vom $\mathrm{N}$. vagus allein und letztere vom N. splanchnicus allein kontrolliert werden.

Aus der Versuchsreihe in diesem Kapitel können wir mit Sicherbeit Folgendes schliessen : N. vagus befördert die innere Sekretion des Pankreas.

\section{Literatur.}

(1) S. Ukai, Tohoku-Igaku-Zasshi 1925, 9, 37.

(2) Eppinger, Falta u. Rudinger, Zeitschr. f. klin. Med. 1908, 66, 1.

(3) Jo sé M. de Corall, Zeitschr. f. Biol., 1918, 68, 395.

(4) R. Macleod, Brit. Med. Journ., 1923, Nr. 3266, 165.

(5) Me Cormick, R. Macleod und M.K. O'brien, Trans. Roy. Soc. Can. Sect. V, 1923, p. 57.

(6) M. Eigner, Zentralbl. f. Physiol., 1915, 30, 445.

(7) Stewart and Rogoff, Zit. nach McCormick u. a. (4).

(8) I. B ang, Mikromethoden zur Blutuntersuchung, Wiesbaden 1922, S. 35.

(9) T. Hoshi, diese Nr. dies. Zeitschr. 327.

(10) S.L. Baker, F. Dickens und D.E. Dodds, Brit. Journ. Exp. Path., 1924, 5,

(11) Tscheboksaroff, Pflüger's Arch. 1910, 137, 187.

(12) Asher, Zeitschr. f. Biol, 1911, 58, 274.

(13) Bied I, Innere Sekretion, 3. Aufl., 2. Teil, Berlin \& Wien, 1916, S. 8.

(14) Elliott, Journ. of Physiol., 1913, 46, 285.

(15) T. Hos hi, erscheint in „Mitteil. über Allg. Path. u. Path. Anat. Bd. III, Heft 2."

Die Untersuchung wurde mit Unterstützung der Sai to-Hōonkwaì Stiftung ausgeführt, der ich hiermit bestens dafür danke.

Prof. T. Kumagai. 